Supporting Information for:

\title{
Nonionic Surfactant Properties of Amphiphilic Hyperbranched Polyglycerols
}

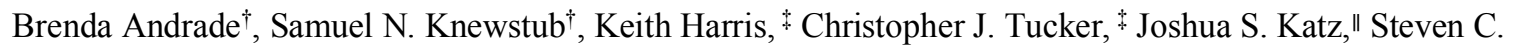 \\ Zimmerman $^{\dagger *}$ \\ 'Department of Chemistry, University of Illinois at Urbana-Champaign, Urbana, Illinois 61801, United States \\ ${ }^{*}$ Formulation Science, Corporate Research and Development, The Dow Chemical Company, Midland, Michigan \\ 48667, United States \\ "Pharma Solutions, DuPont Nutrition \& Biosciences, Wilmington, Delaware 19803, United States
}

*Author to whom correspondence should be addressed

(sczimmer@illinois.edu)

Number of pages: 22

Number of figures: 38

\section{$\underline{\text { Table of Contents }}$}

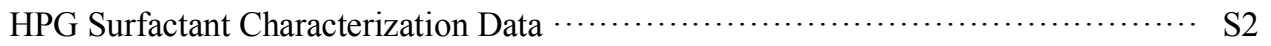

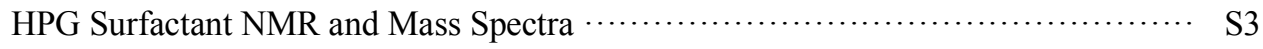

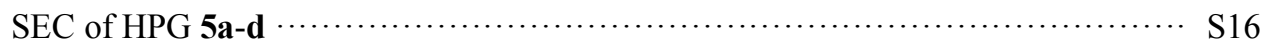

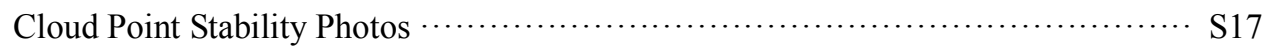

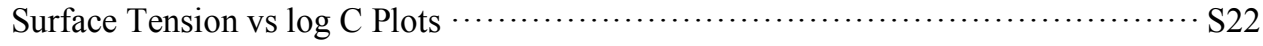


HPG $1{ }^{1} \mathrm{H}$ NMR $\left(500 \mathrm{MHz}, \mathrm{CD}_{3} \mathrm{OD}\right) \delta, 3.91-3.57(\mathrm{~m}, 139 \mathrm{H}), 1.57(\mathrm{t}, 2 \mathrm{H}), 1.33(\mathrm{~m}, 6 \mathrm{H}), 0.91(\mathrm{bt}, 3 \mathrm{H}){ }^{13} \mathrm{C}$ NMR $(126$ $\left.\mathrm{MHz}, \mathrm{CD}_{3} \mathrm{OD}\right) \delta$ 79.87, 73.91, 72.22, 64.40. MALDI-TOF 2051.1 (M+ Na+) 1059.7 (M+ initiator)

HPG $2{ }^{1} \mathrm{H}$ NMR $\left(500 \mathrm{MHz}, \mathrm{CD}_{3} \mathrm{OD}\right) \delta 4.56(\mathrm{~s}, 35 \mathrm{H}), 3.99-3.36(\mathrm{~m}, 152 \mathrm{H}), 1.57(\mathrm{~s}, 3 \mathrm{H}), 1.28(\mathrm{~s}, 14 \mathrm{H}), 0.89(\mathrm{t}, J=$ $6.7 \mathrm{~Hz} .3 \mathrm{H}){ }^{13} \mathrm{C}$ NMR $\left(101 \mathrm{MHz}, \mathrm{CD}_{3} \mathrm{OD}\right) \delta 80.21,80.01,78.61,72.68,71.01,69.50,69.46,63.14,29.56$. MALDITOF $467.3\left(\mathrm{C}_{12} \mathrm{H}_{25} \mathrm{O}-\mathrm{Gly}_{4}: \mathrm{M}^{+}\right) 698.3\left(\mathrm{M}^{+}\right.$- initiator)

HPG $3{ }^{1} \mathrm{H}$ NMR $\left(500 \mathrm{MHz}, \mathrm{CD}_{3} \mathrm{OD}\right) \delta 3.92-3.51(\mathrm{~m}, 3651 \mathrm{H}), 1.51(\mathrm{bs}, 1 \mathrm{H}), 1.28(\mathrm{~m}, 28 \mathrm{H}), 0.89(\mathrm{t}, J=6.8 \mathrm{~Hz} .3 \mathrm{H})$. ${ }^{13} \mathrm{C}$ NMR $\left(101 \mathrm{MHz}, \mathrm{CD}_{3} \mathrm{OD}\right) \delta 80.03,77.16,75.10,73.03,70.99,69.36,68.33,63.11,61.34$. MALDI-TOF 1096.3 $\left(\mathrm{C}_{20} \mathrm{H}_{41} \mathrm{O}-\mathrm{Gly}_{11} \mathrm{M}^{+}\right) 614.9\left(\mathrm{M}^{+}\right.$- initiator $)$

HPG $4{ }^{1} \mathrm{H}$ NMR $\left(500 \mathrm{MHz}, \mathrm{CD}_{3} \mathrm{OD}\right) \delta 3.93-3.60(m, 2273 \mathrm{H}), 1.57(m, 2 \mathrm{H}) 1.34(\mathrm{~m}, 31 \mathrm{H}), 0.93(\mathrm{~m}, 6 \mathrm{H}) .{ }^{13} \mathrm{C}$ NMR (126 MHz, MeOD) $\delta 80.24,80.02,78.47,72.17,71.55,71.06,70.83,69.59,69.29,63.08,62.98,61.42,60.86,14.05$ MALDI-TOF $815.8\left(\mathrm{M}^{+}\right.$- initiator), $964.0\left(\mathrm{M}^{+}\right) 986.0\left(\mathrm{M}+\mathrm{Na}^{+}\right.$- initiator)

HPG 5a ${ }^{1} \mathrm{H}$ NMR $(500 \mathrm{MHz}, \mathrm{MeOD}) \delta 3.94-3.61(m, 940 \mathrm{H}), 1.6-1.2(m, 26 \mathrm{H}), 0.93(m, 6 \mathrm{H}) .{ }^{13} \mathrm{C}$ NMR $(126 \mathrm{MHz}$, MeOD) $\delta 80.12,80.02,78.74,78.47,72.58,71.53,71.06,70.83,69.57,68.93,63.07,61.42$. MALDI-TOF 1282.7 $\left(\mathrm{M}+\mathrm{Na}^{+}\right.$- initiator), $1299.2\left(\mathrm{M}+\mathrm{Na}^{+}\right)$

HPG 5b ${ }^{1} \mathrm{H}$ NMR $(500 \mathrm{MHz}, \mathrm{MeOD}) \delta$ 4.1-3.4 $(m, 4772 \mathrm{H}), 2.25-1.75(m, 9 \mathrm{H}), 1.55-1.70(m, 29 \mathrm{H}), 0.87(m, 6 \mathrm{H})$ ${ }^{13} \mathrm{C}$ NMR (126 MHz, MeOD) $\delta$ 80.24, 80.03, 78.75, 78.47, 72.53, 71.54, 71.07, 70.83, 69.58, 69.29, 63.08, 61.41 . MALDI-TOF $763.3\left(\mathrm{M}+\mathrm{Na}^{+}\right.$- initiator), $779.3\left(\mathrm{M}^{+}\right)$

HPG 5c ${ }^{1} \mathrm{H}$ NMR $(500 \mathrm{MHz}, \mathrm{MeOD}) \delta 4.00-3.37(m, 2890 \mathrm{H}), 2.25-1.83(m, 4 \mathrm{H})$ 1.69-1.10 $(m, 26 \mathrm{H}) 0.93(m, 6 \mathrm{H})$. ${ }^{13} \mathrm{C}$ NMR (126 MHz, MeOD) $\delta$ 80.23, 80.02, 78.73, 78.45, 72.54, 71.52, 71.04, 70.81, 69.57, 69.27, 63.06, 61.03. MALDI-TOF $985.4\left(\mathrm{M}+\mathrm{Na}^{+}\right.$- initiator), $1001.4\left(\mathrm{M}+\mathrm{Na}^{+}\right)$

HPG 5d ${ }^{1} \mathrm{H}$ NMR $(500 \mathrm{MHz}, \mathrm{MeOD}) \delta 4.11-3.40(m, 153 \mathrm{H}) 1.68-1.30(m, 24 \mathrm{H}), 0.96(m, 6 \mathrm{H}) .{ }^{13} \mathrm{C}$ NMR $(126$ $\mathrm{MHz}, \mathrm{MeOD}) \delta 80.19,79.95,78.42,75.86,74.59,72.53,71.51,70.85,69.53,69.27,67.87,67.73,66.92,62.99$, $61.62,60.86,60.20,33.60,31.70,29.10,25.06,22.38,13.21$. MALDI-TOF $837.4\left(\mathrm{M}+\mathrm{Na}^{+}\right.$- initiator), $853.3(\mathrm{M}+$ $\left.\mathrm{Na}^{+}\right)$

HPG $6{ }^{1} \mathrm{H}$ NMR $\left(500 \mathrm{MHz}, \mathrm{CD}_{3} \mathrm{OD}\right) \delta 3.92-3.52(\mathrm{~m}, 940 \mathrm{H}), 1.70-0.91(\mathrm{~m}, 36 \mathrm{H}) .{ }^{13} \mathrm{C} \mathrm{NMR}\left(126 \mathrm{MHz}, \mathrm{CD}_{3} \mathrm{OD}\right) \delta$ 74.87, 73.85, 72.11, 71.49, 70.57, 64.38, 26.27 MALDI-TOF 1504.5 $\left(\mathrm{M}^{+}\right)$. 


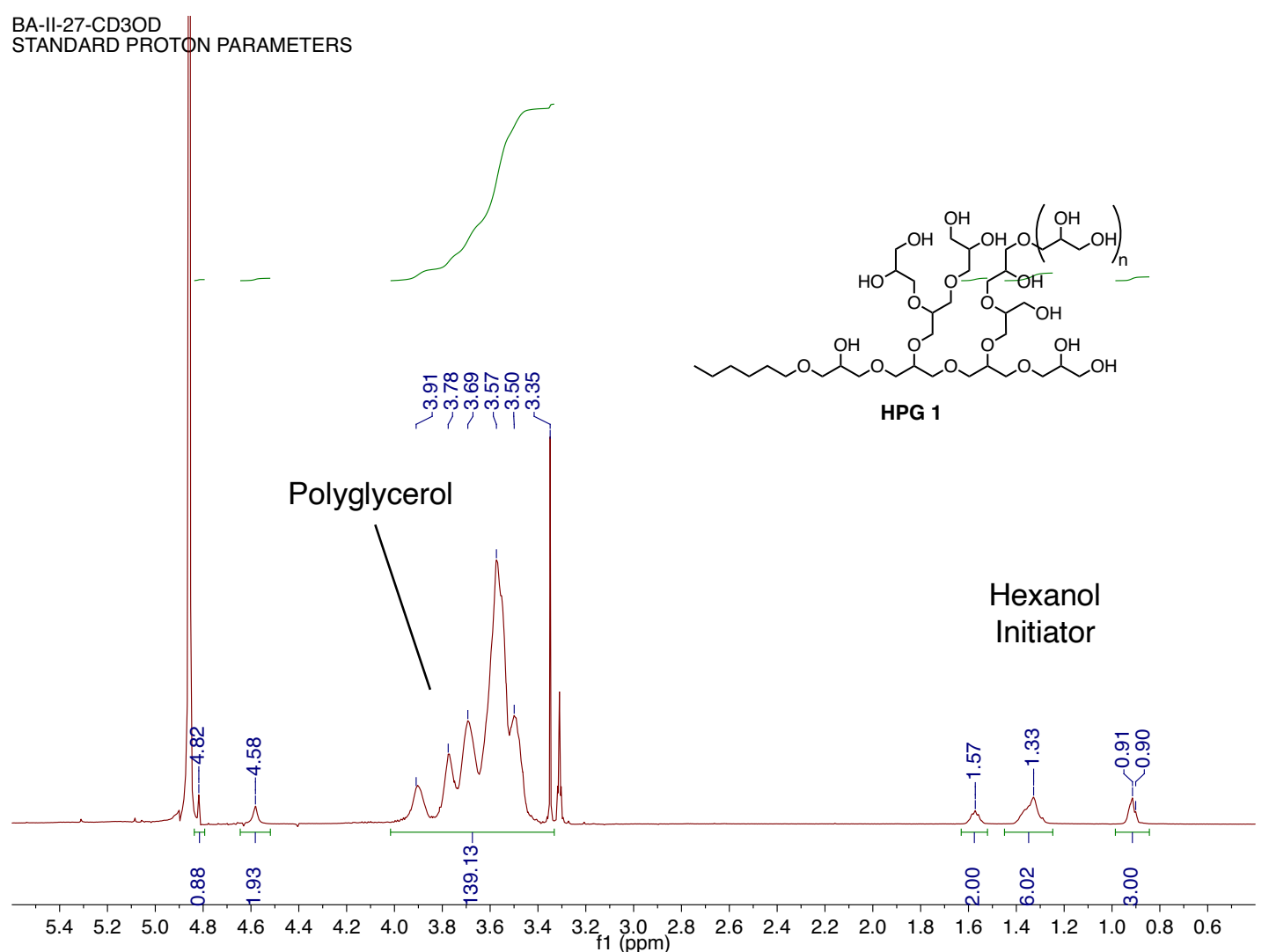

Figure S1. ${ }^{1} \mathrm{H}$ NMR $400 \mathrm{MHz}$, Methanol- $d_{4}$ of HPG 1

13CNMR-BA-II-27-CD3OD-Crude
STANDARD CARBON PARAMETERS

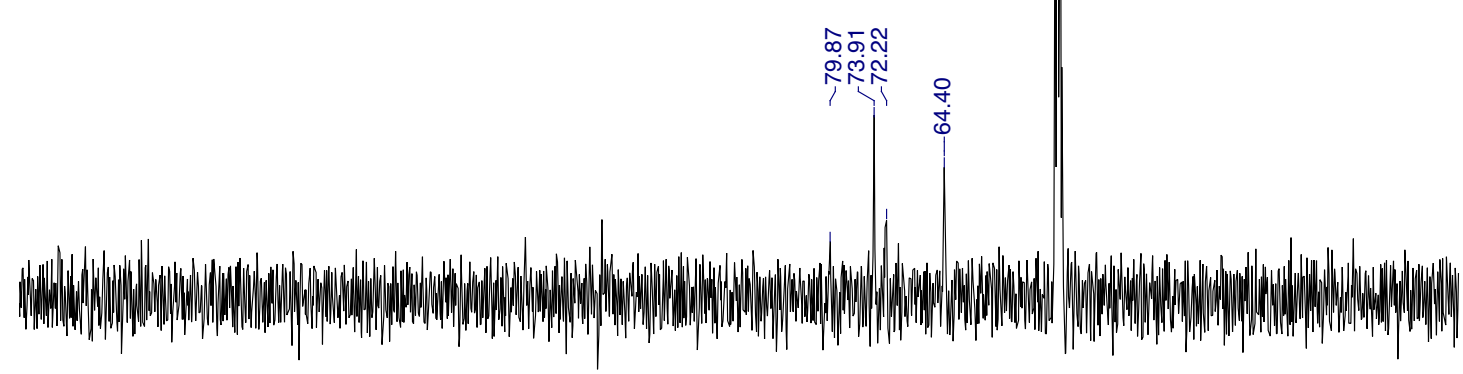

$\begin{array}{llllllllll}180 & 170 & 160 & 150 & 140 & 130 & 120 & 110 & 100 \begin{array}{c}90 \\ \mathrm{f} 1(\mathrm{ppm})\end{array}\end{array}$

Figure S2. ${ }^{13} \mathrm{C}$ NMR $500 \mathrm{MHz}$, Methanol- $d_{4}$ of HPG 1 


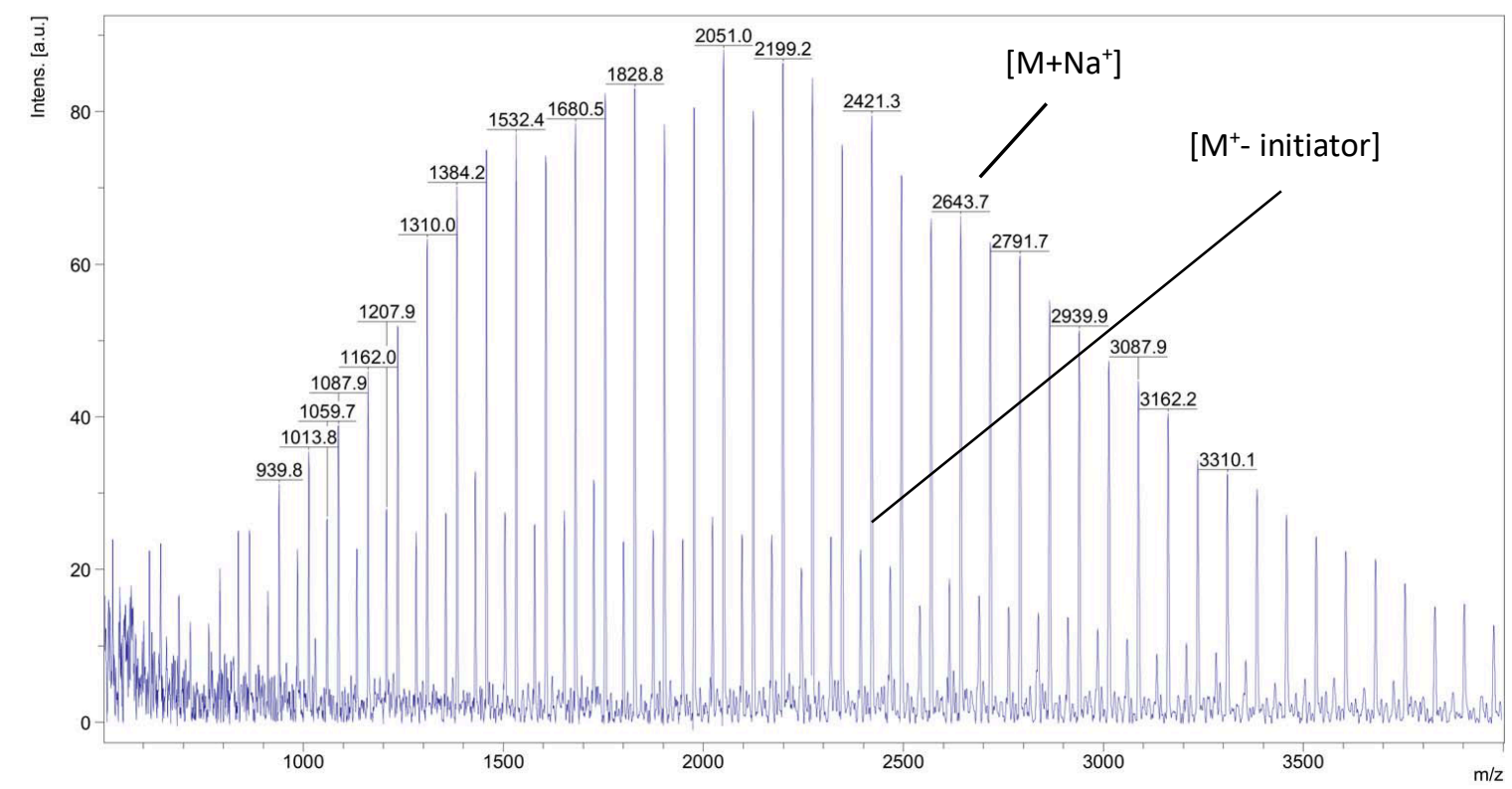

Figure S3. MALDI-TOF Mass Spectra of HPG 1

1-SK-43-F4_CD3OD_1H_Unlin425HPG STANDARD PROTON PARAMETERS

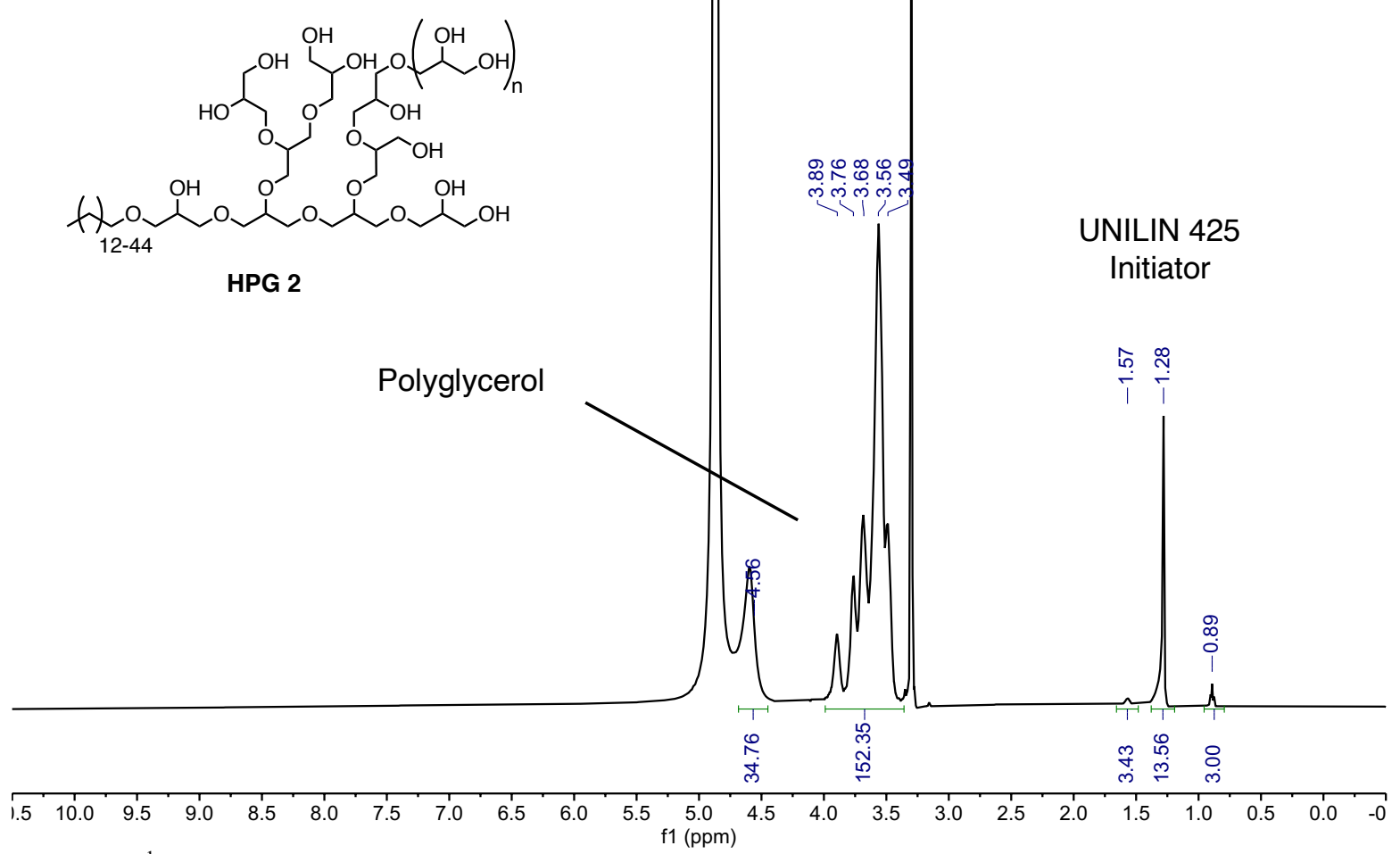

Figure S4. ${ }^{1} \mathrm{H}$ NMR $400 \mathrm{MHz}$, Methanol- $d_{4}$ of HPG 2 


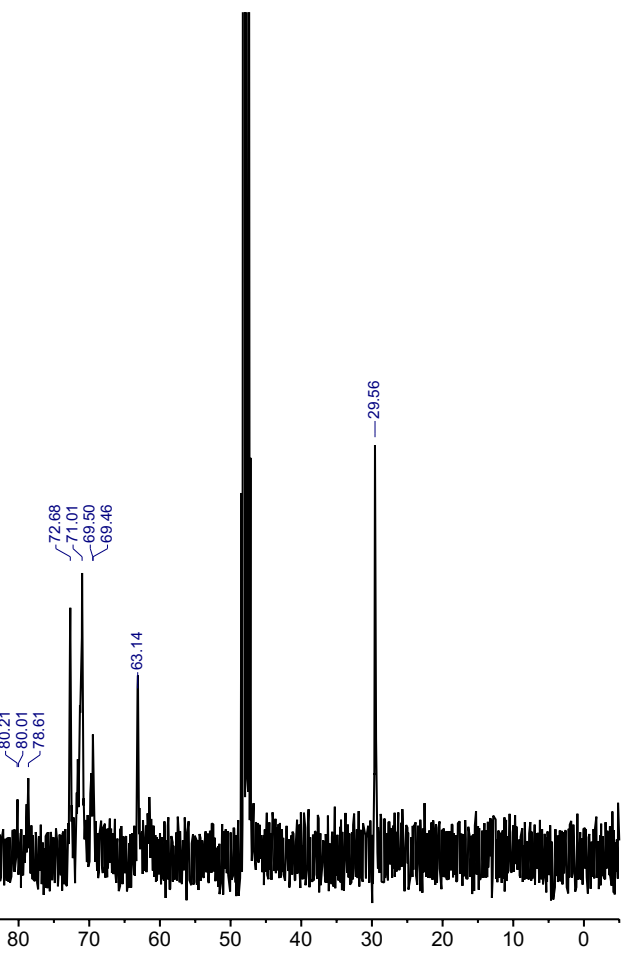

Figure S5. ${ }^{13} \mathrm{C}$ NMR $400 \mathrm{MHz}$, Methanol- $d_{4}$ of HPG 2

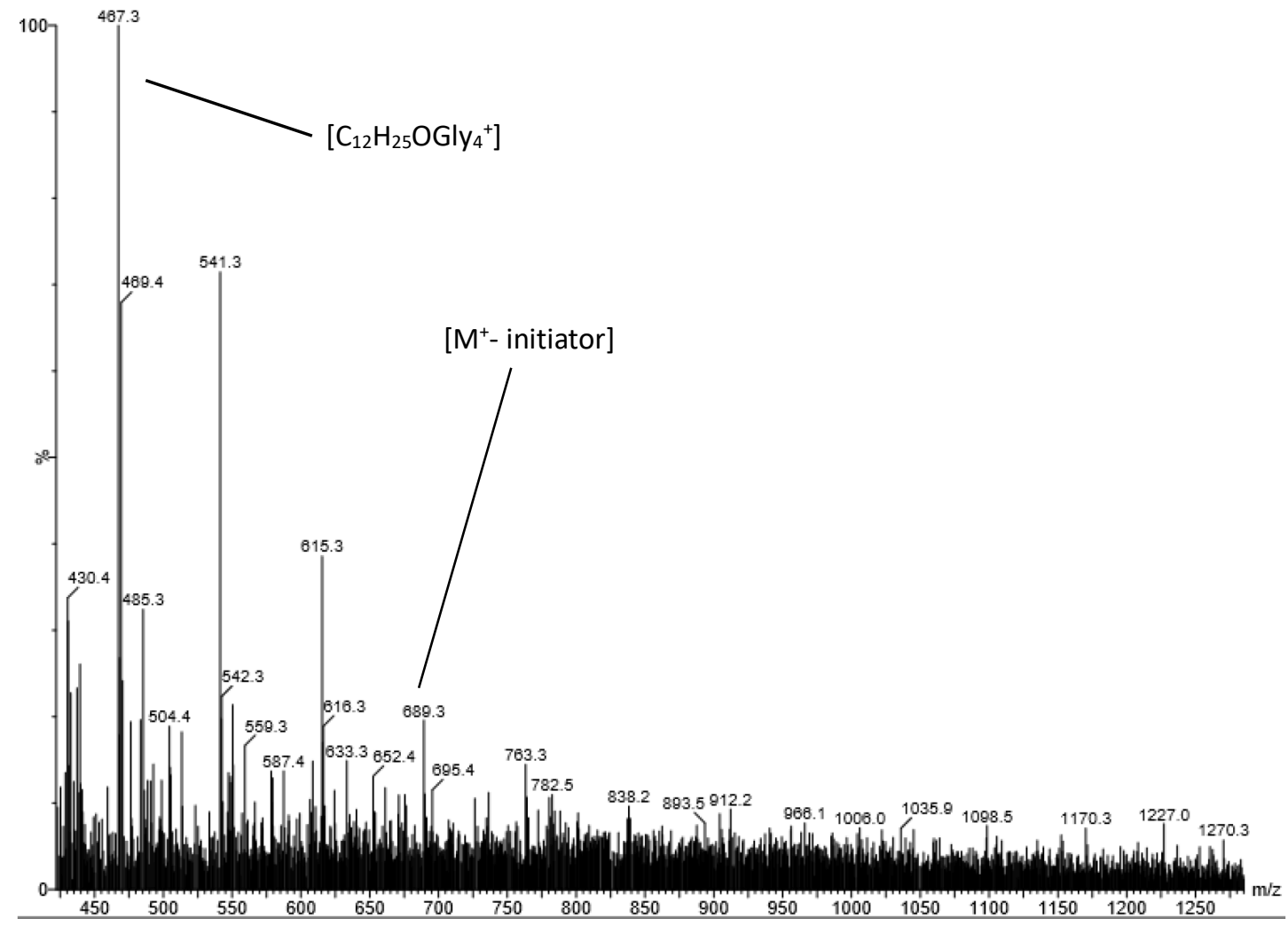

Figure S6. Low-Res ESI-Mass Spectra of HPG 2 


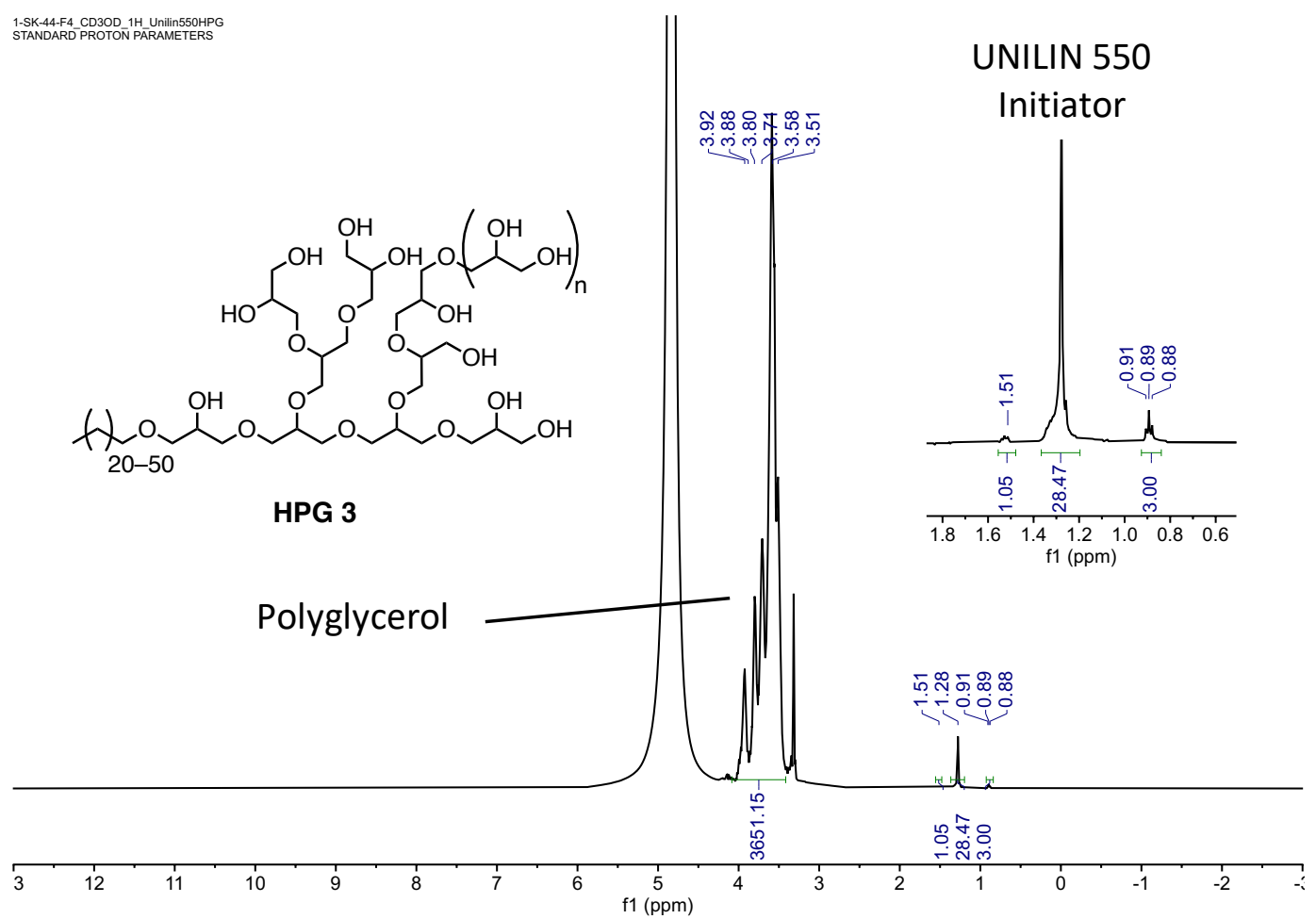

Figure S7. ${ }^{1} \mathrm{H}$ NMR $400 \mathrm{MHz}$, Methanol- $d_{4}$ of HPG 3

1-SK-44-F4_CD3OD_C13_UNILIN550 13C OBSERVE

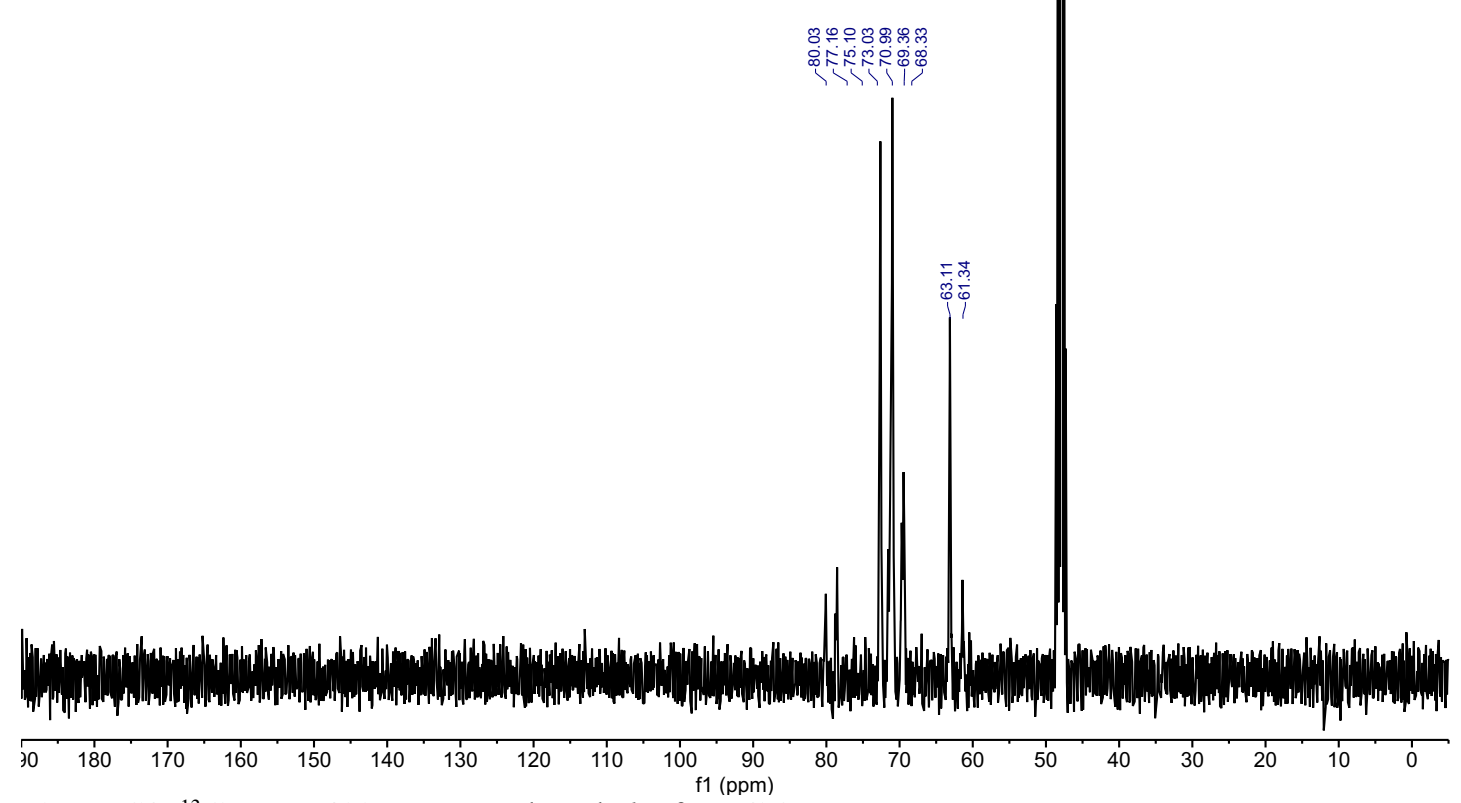

Figure S8. ${ }^{13} \mathrm{C}$ NMR $400 \mathrm{MHz}$, Methanol- $d_{4}$ of HPG 3 


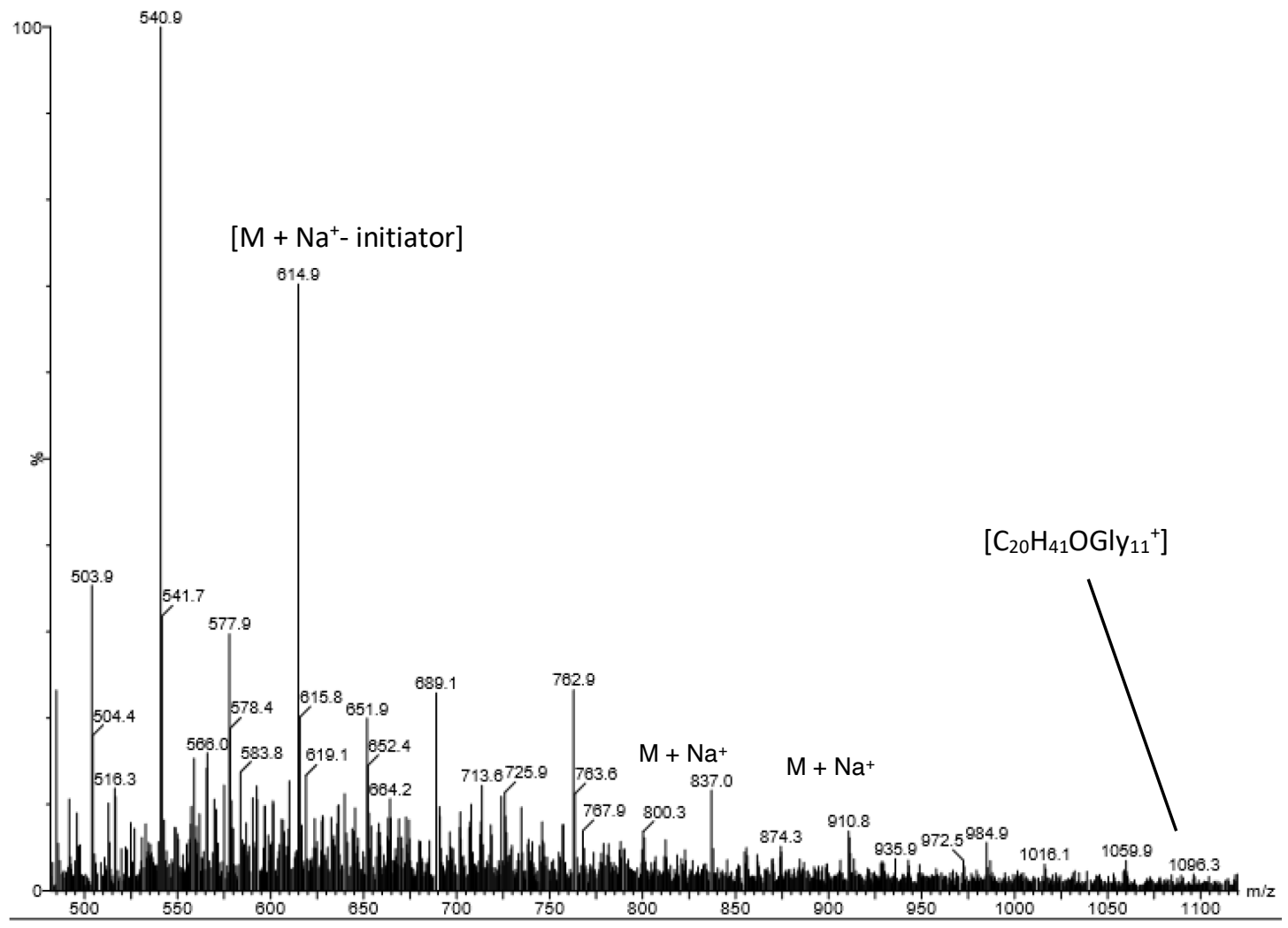

Figure S9. Low-Res ESI-Mass Spectra HPG 3

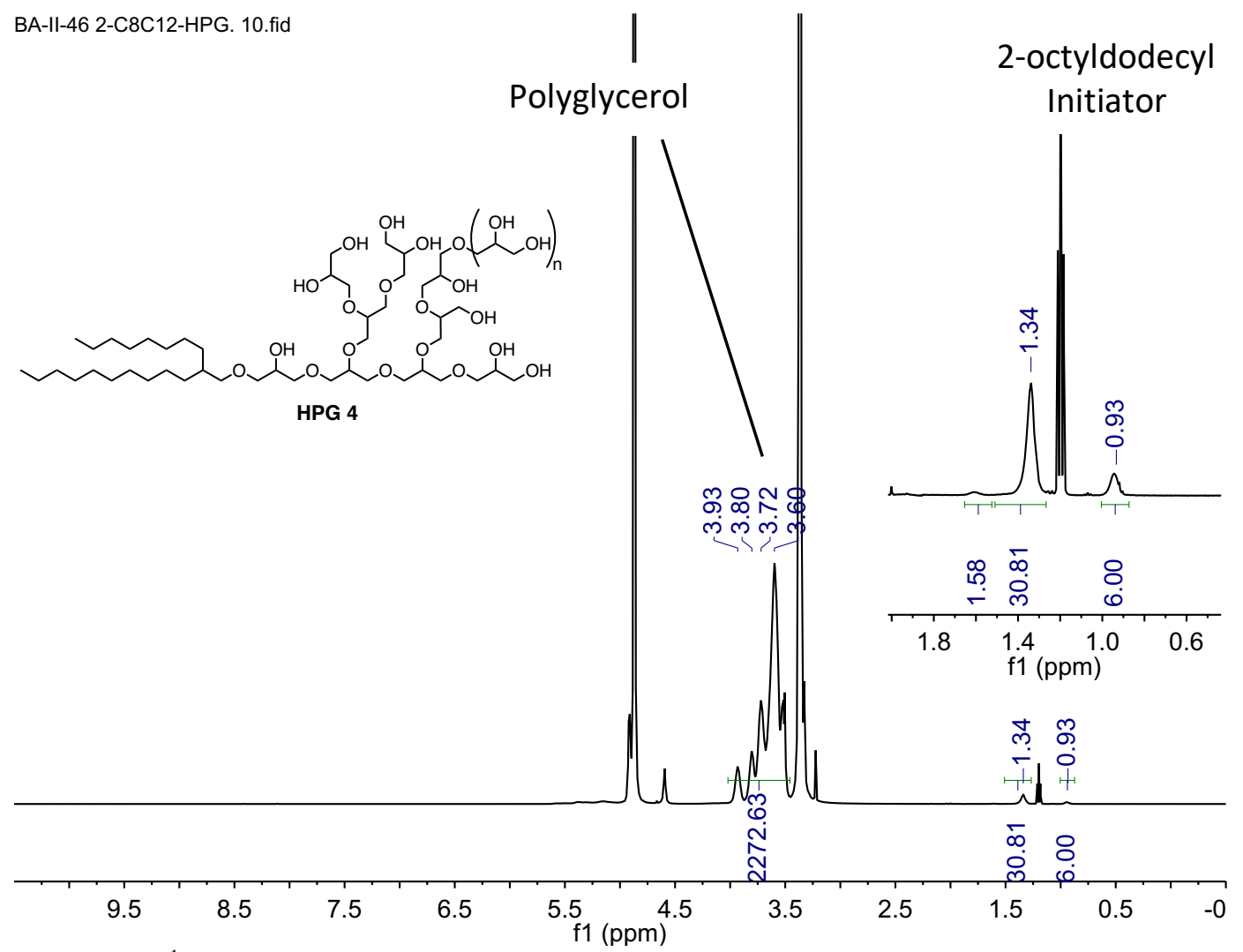

Figure S10. ${ }^{1} \mathrm{H}$ NMR $400 \mathrm{MHz}$, Methanol- $d_{4}$ of HPG 4 


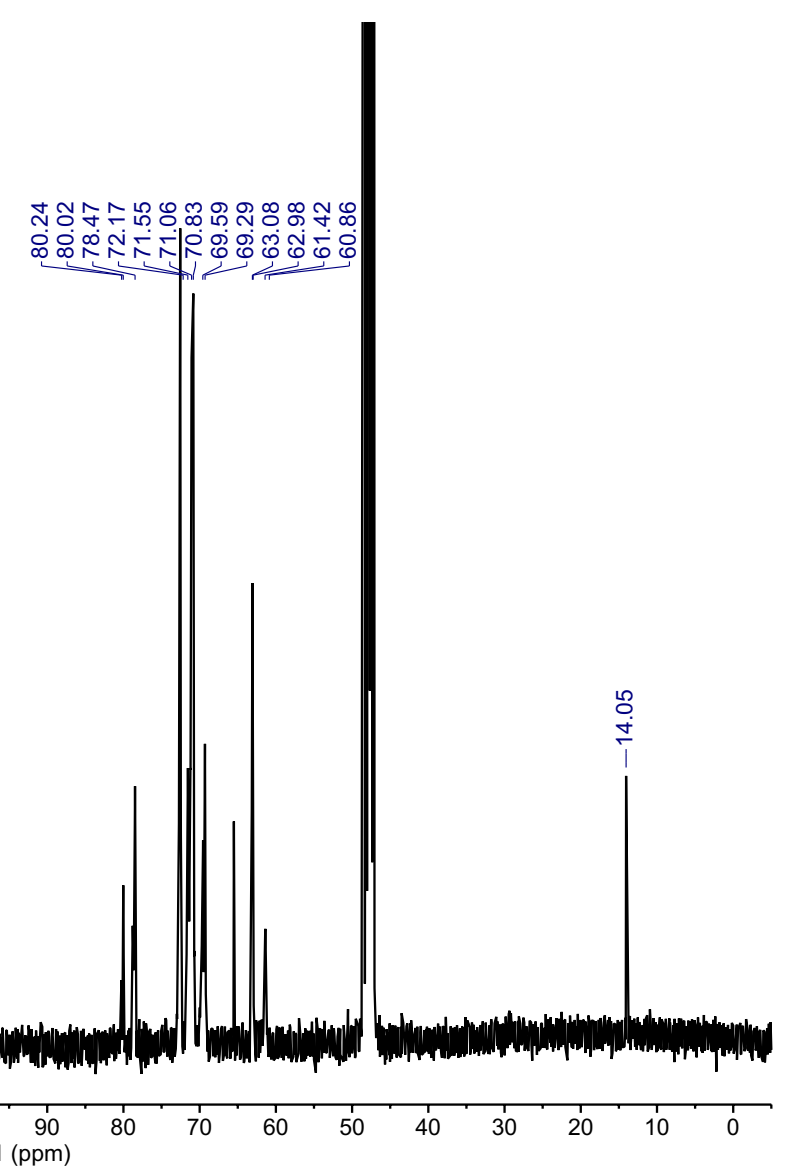

Figure S11. ${ }^{13} \mathrm{C}$ NMR $500 \mathrm{MHz}$, Methanol- $d_{4}$ of HPG 4

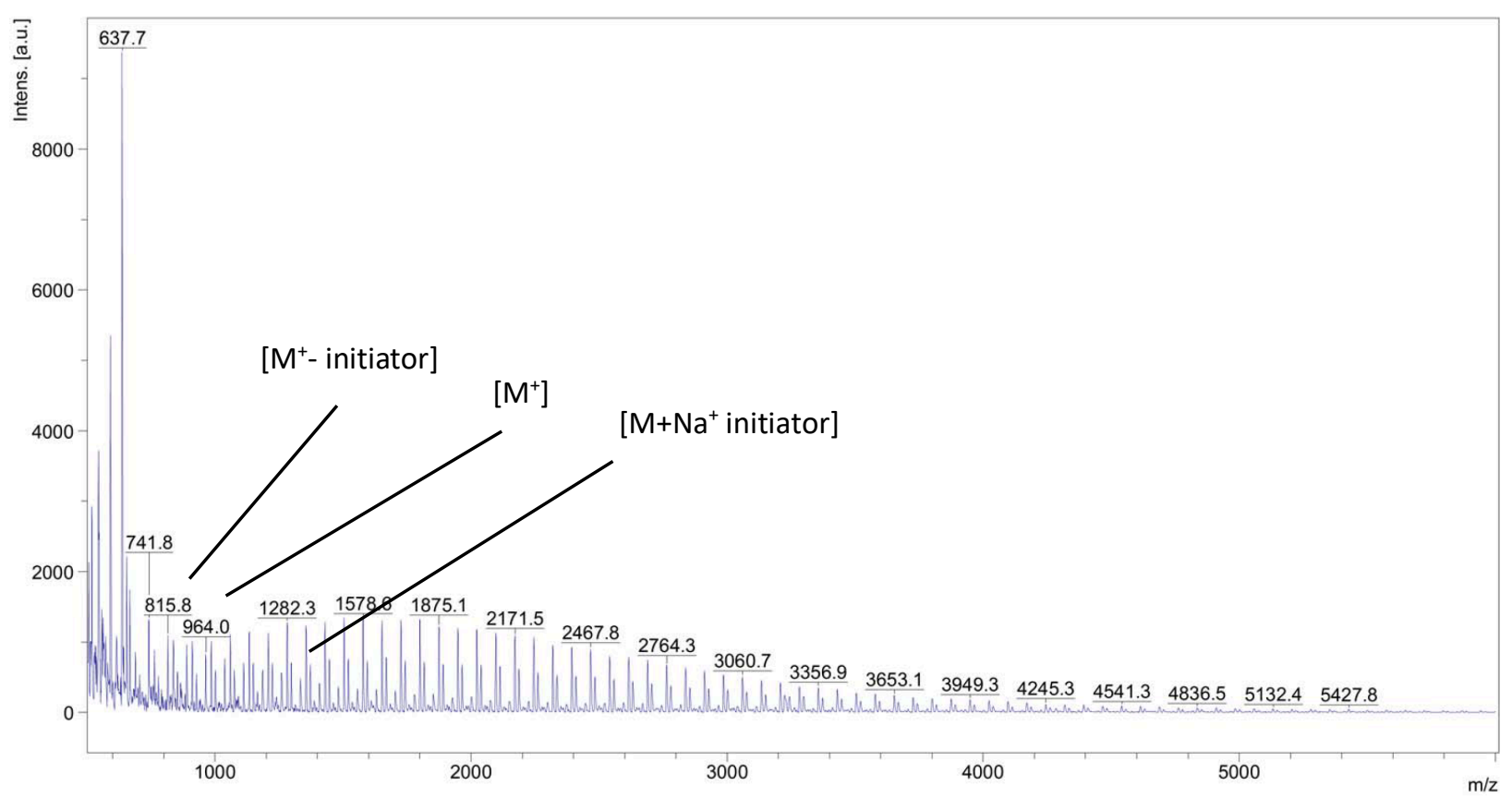

Figure S12. MALDI-TOF Mass Spectra HPG 4 


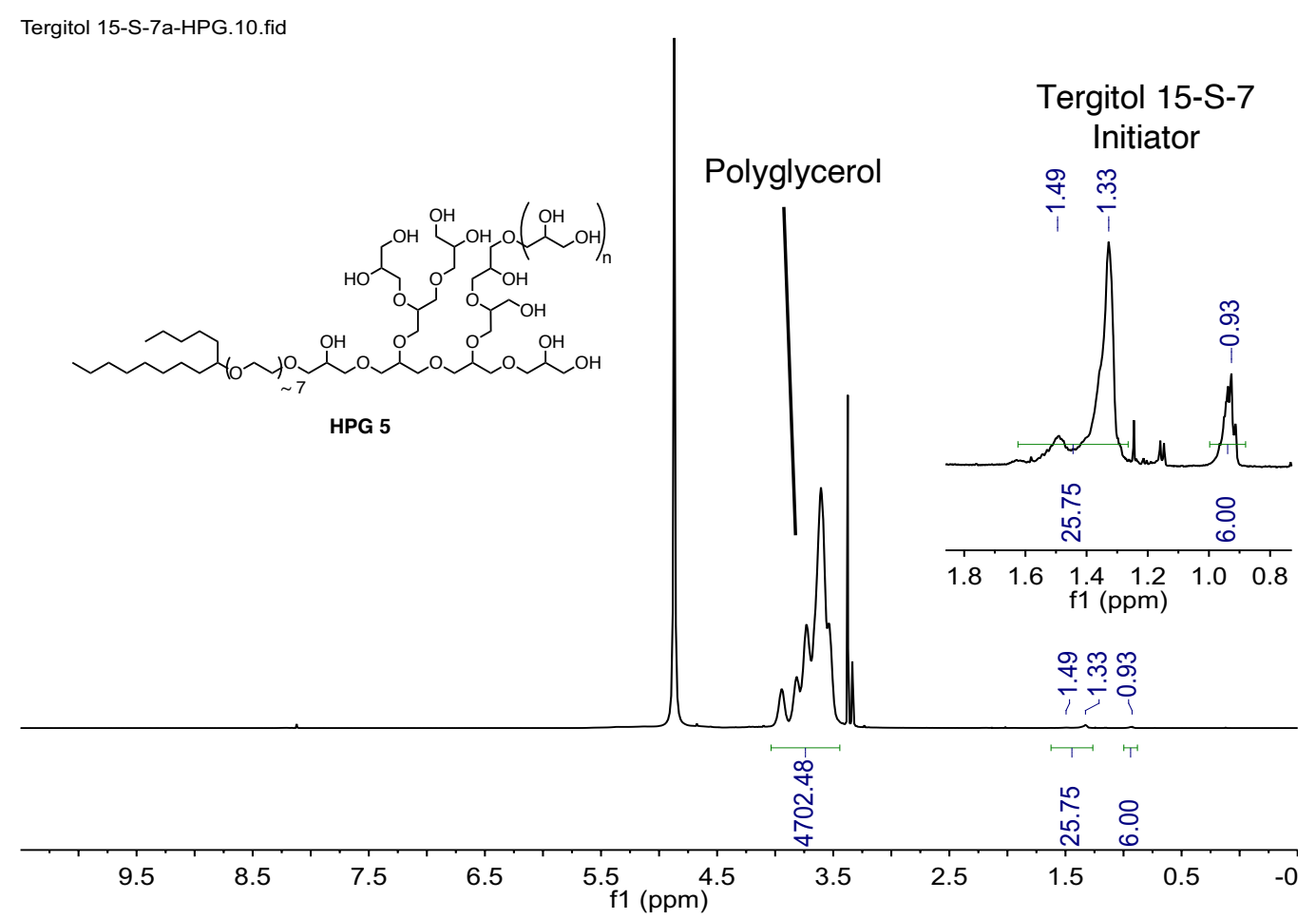

Figure S13. ${ }^{1} \mathrm{H}$ NMR $500 \mathrm{MHz}$, Methanol- $d_{4}$ of HPG 5a

Tergitol 15-S-7a-HPG.11.fid

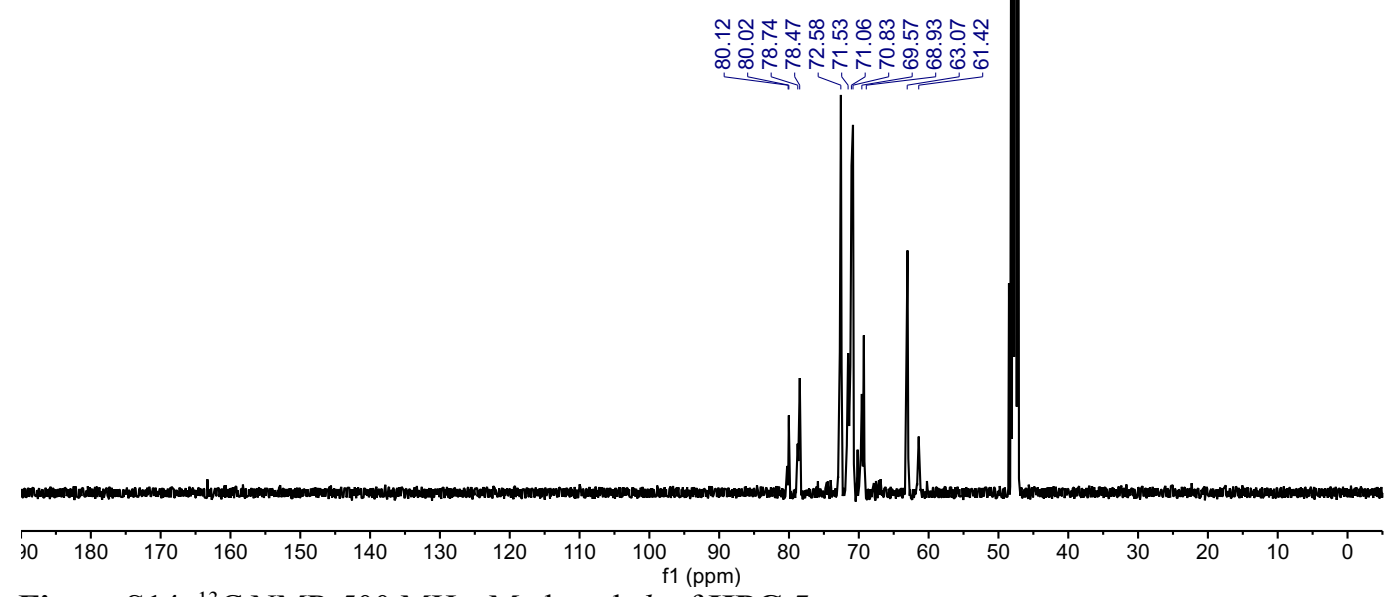

Figure S14. ${ }^{13} \mathrm{C}$ NMR $500 \mathrm{MHz}$, Methanol- $d_{4}$ of HPG 5a 


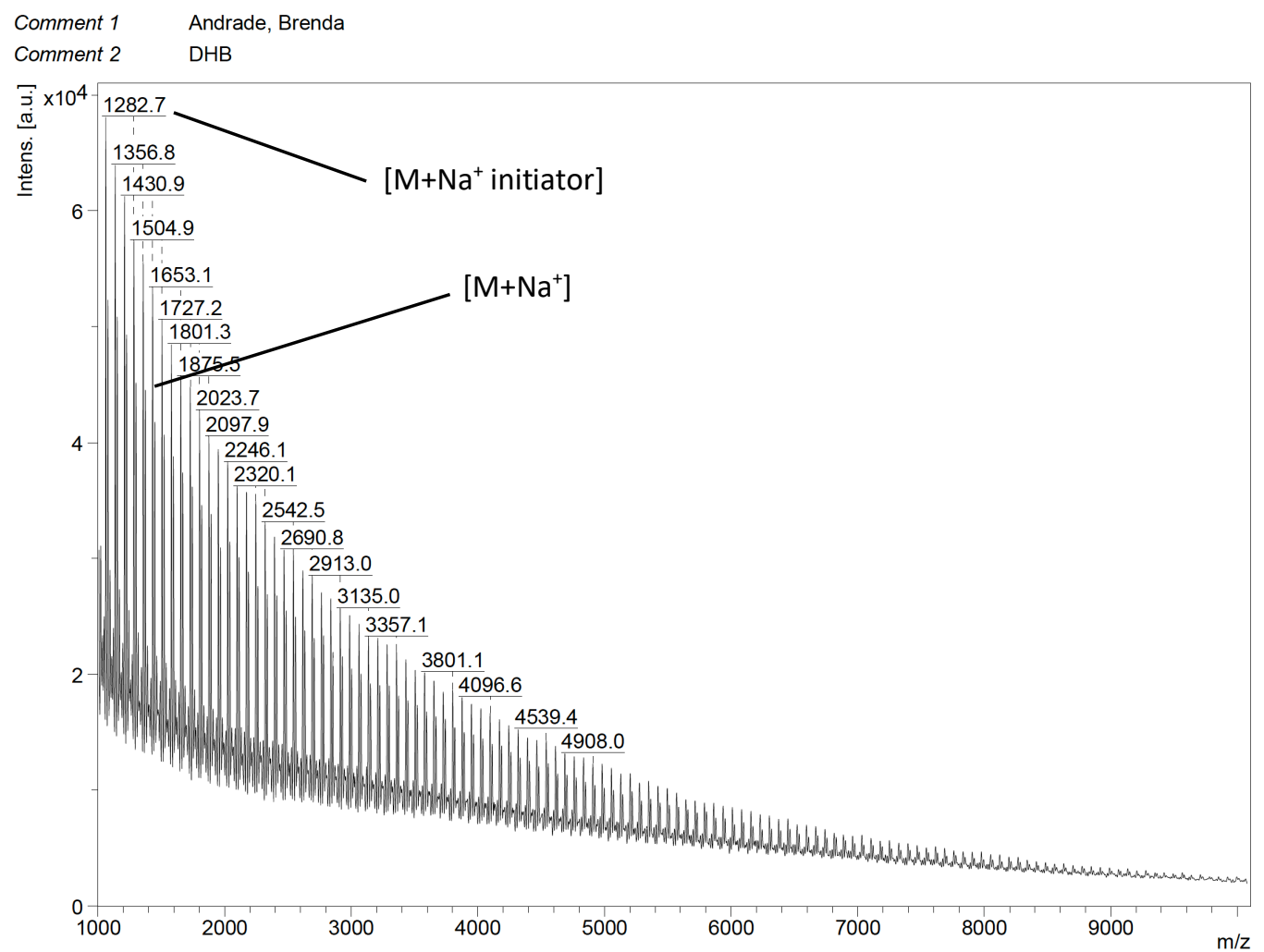

Figure S15. MALDI-TOF Mass Spectra Tergitol HPG 5a

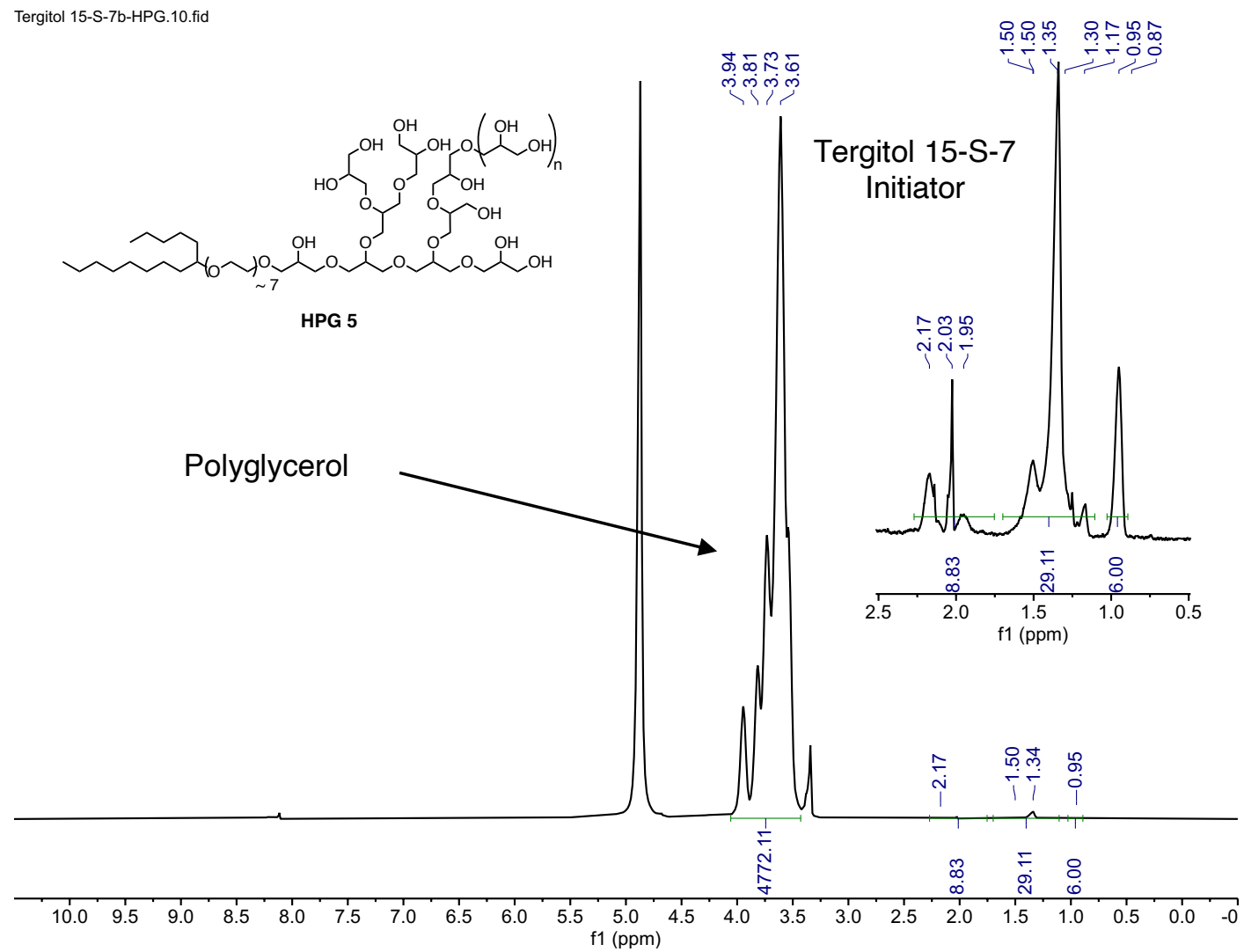

Figure S16. ${ }^{1} \mathrm{H}$ NMR $500 \mathrm{MHz}$, Methanol- $d_{4}$ of HPG 5b 


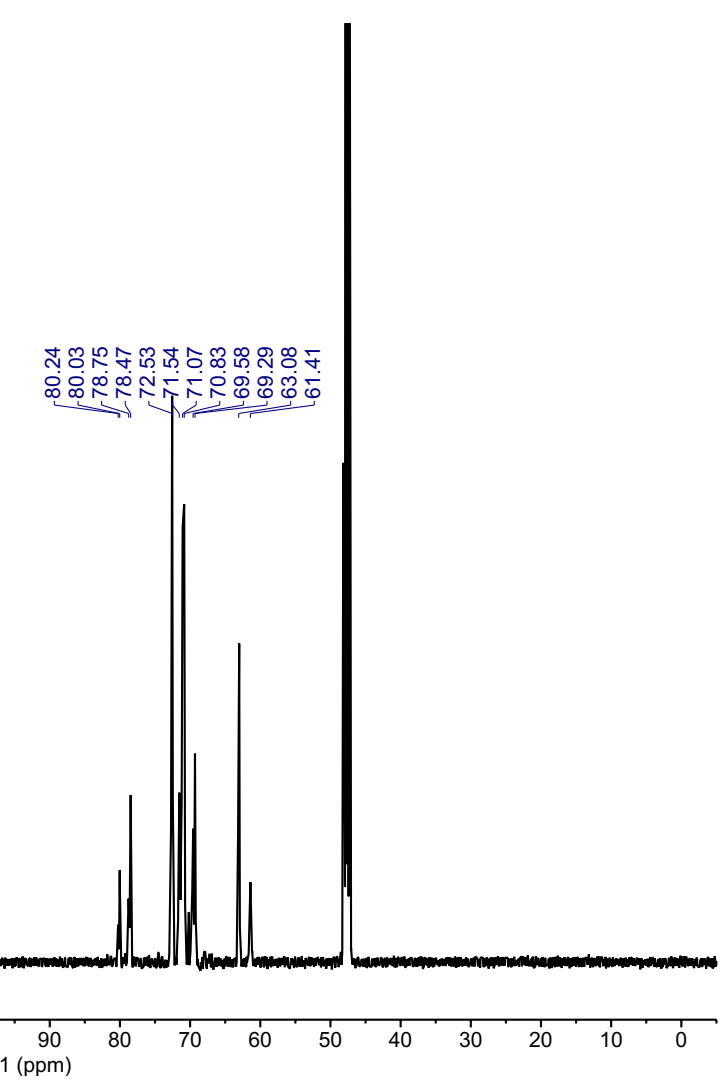

Figure S17. ${ }^{13} \mathrm{C}$ NMR $500 \mathrm{MHz}$, Methanol- $d_{4}$ of HPG 5b

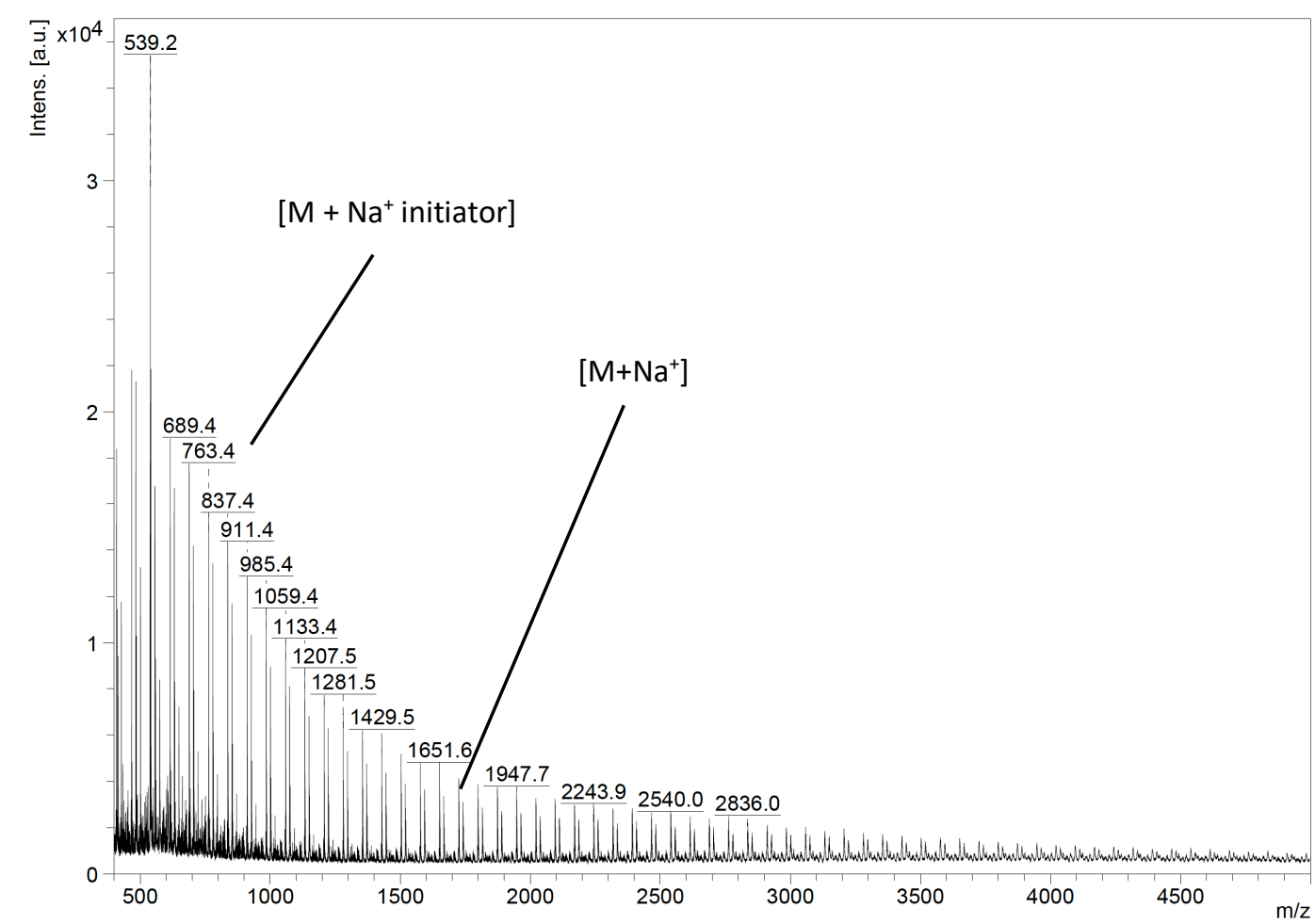

Figure S18. MALDI-TOF Mass Spectra HPG 5b 


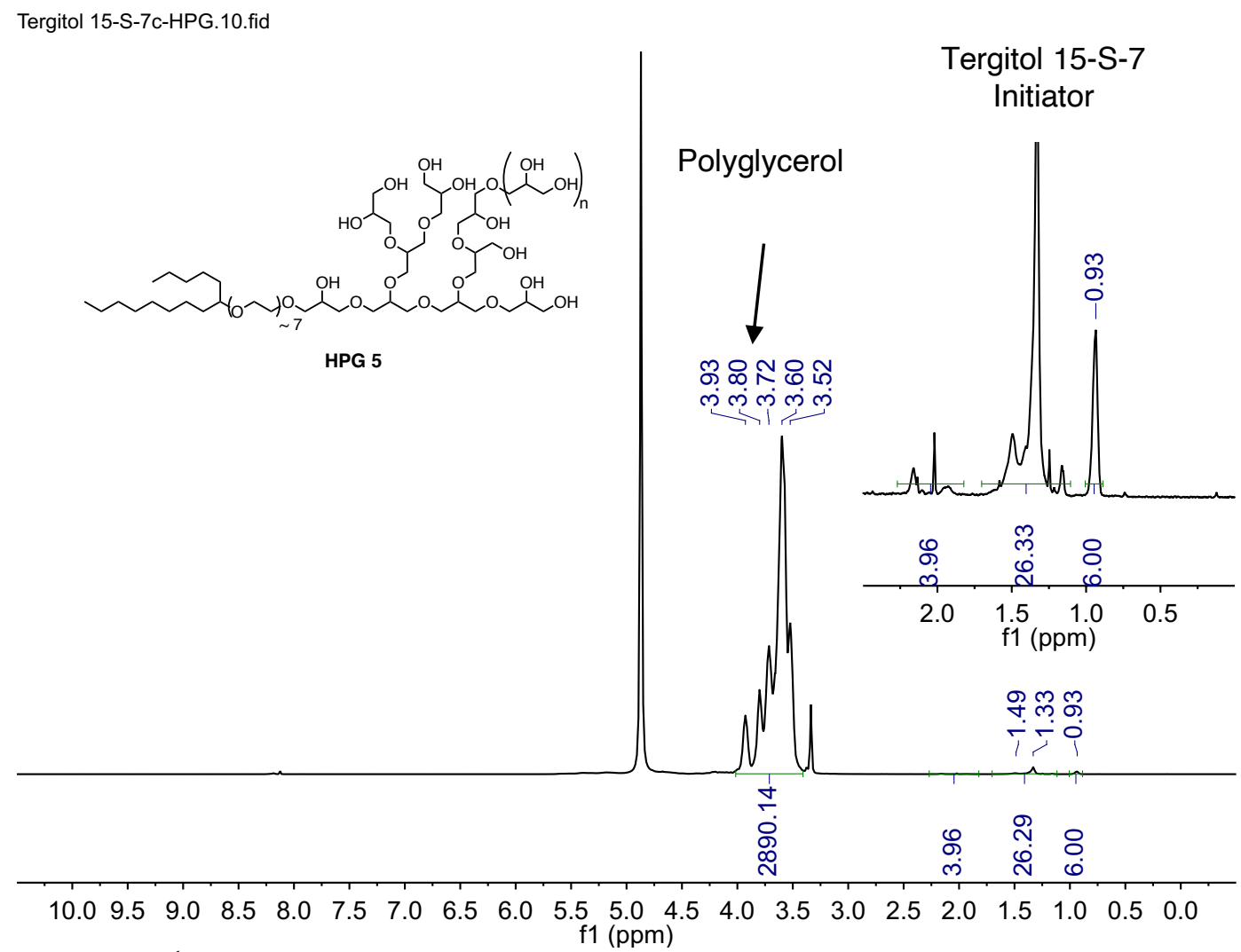

Figure S19. ${ }^{1} \mathrm{H}$ NMR $500 \mathrm{MHz}$, Methanol- $d_{4}$ of HPG 5c Tergitol 15-S-7c-HPG.11.fid

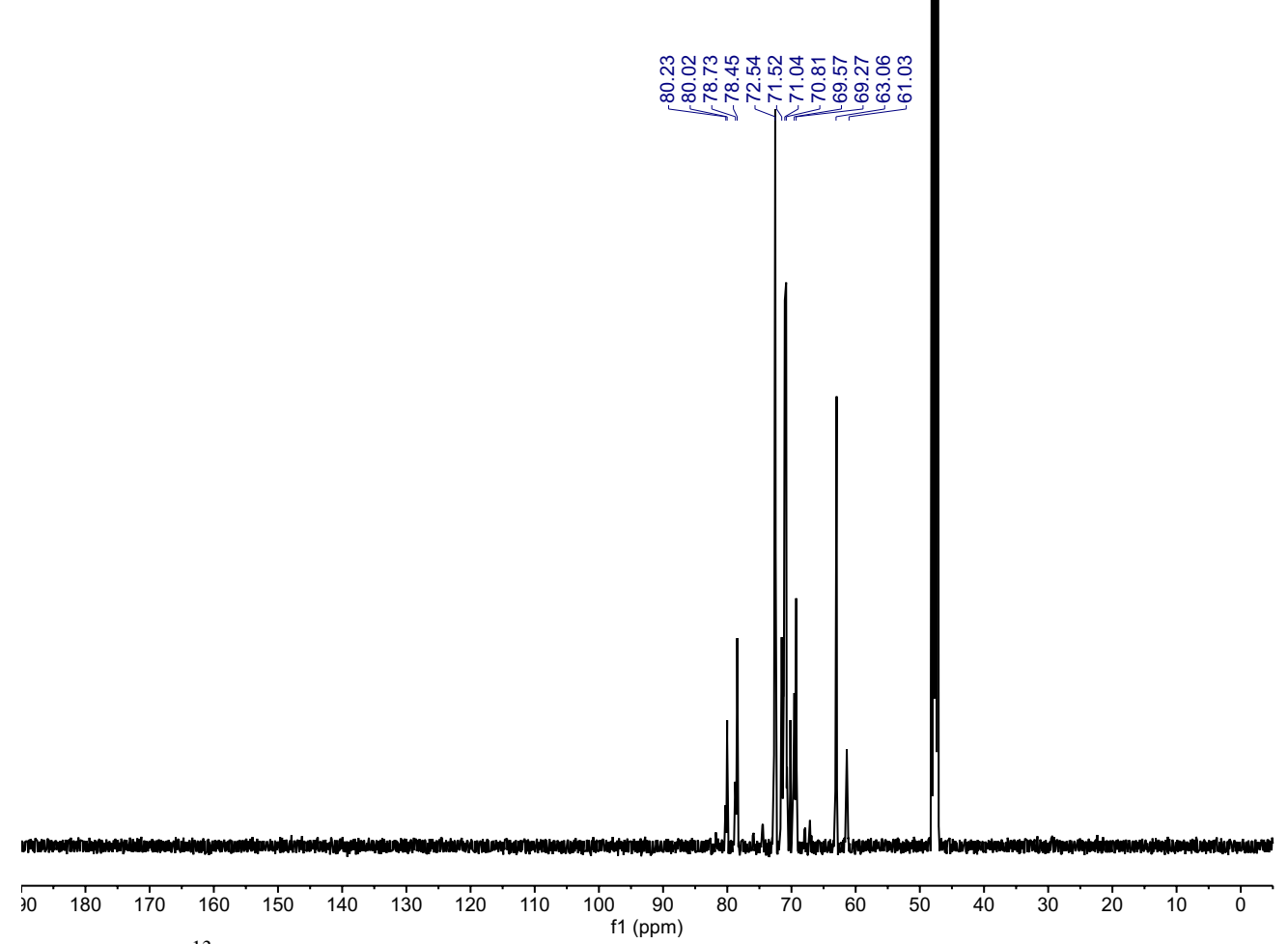

Figure S20. ${ }^{13} \mathrm{C}$ NMR $500 \mathrm{MHz}$, Methanol- $d_{4}$ of HPG 5c 


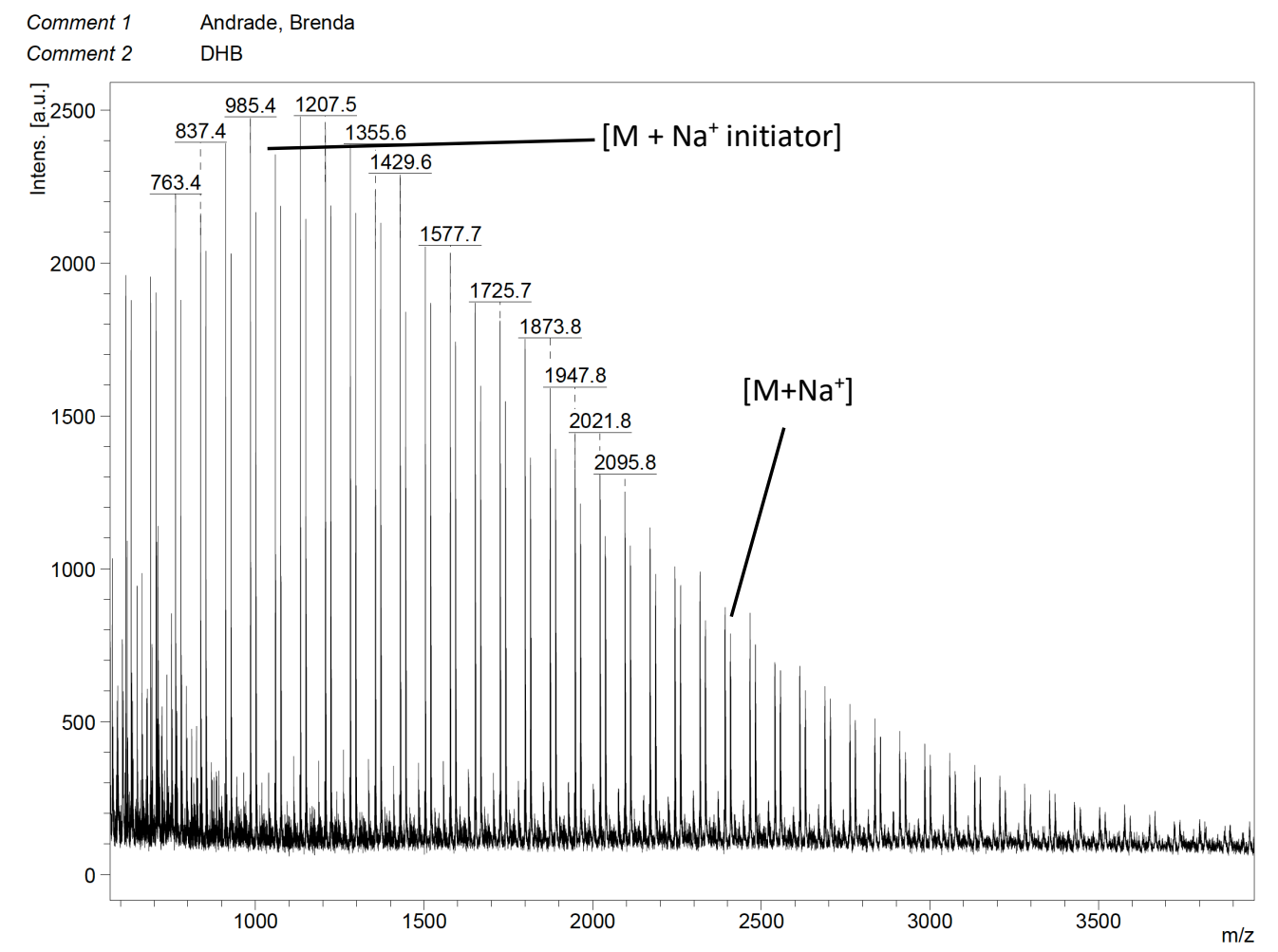

Figure S21. MALDI-TOF Mass Spectra HPG 5c Tergitol 15-S-7d-HPG.10.fid

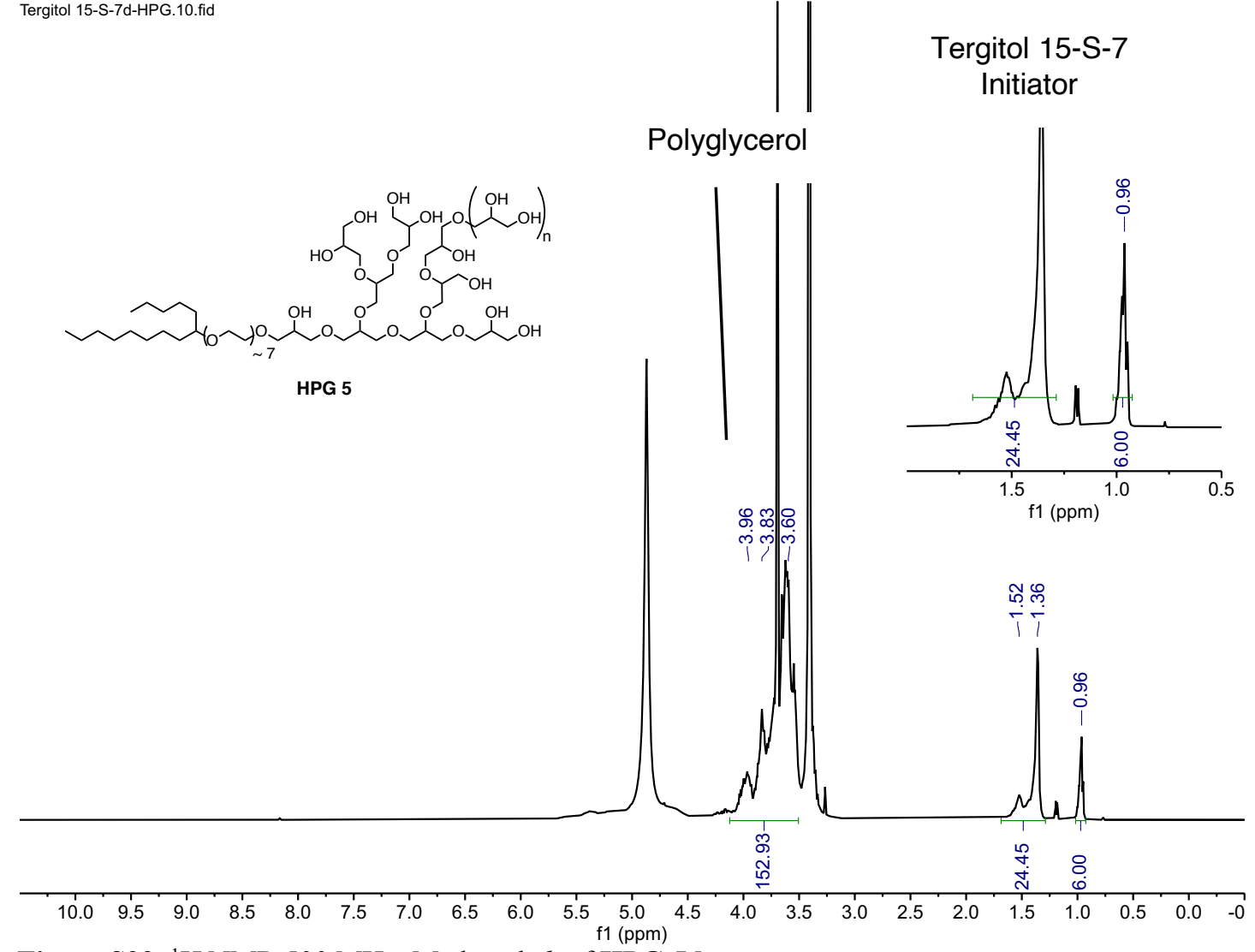

Figure S22. ${ }^{1} \mathrm{H}$ NMR $500 \mathrm{MHz}$, Methanol- $d_{4}$ of HPG 5d 

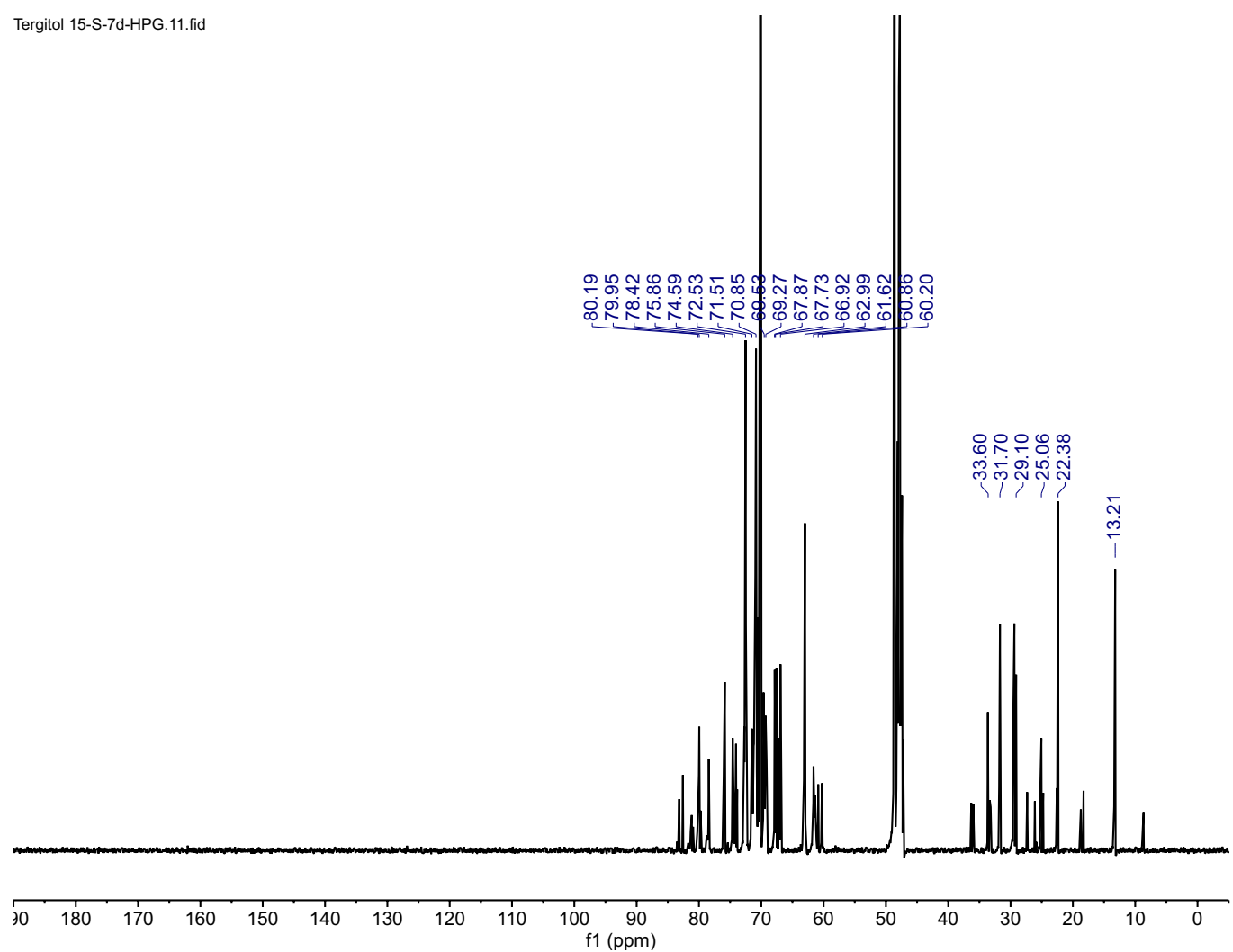

Figure S23. ${ }^{13} \mathrm{C}$ NMR $500 \mathrm{MHz}$, Methanol- $d_{4}$ of HPG 5d

Comment 1 Andrade, Brenda

\section{Comment 2 DHB}

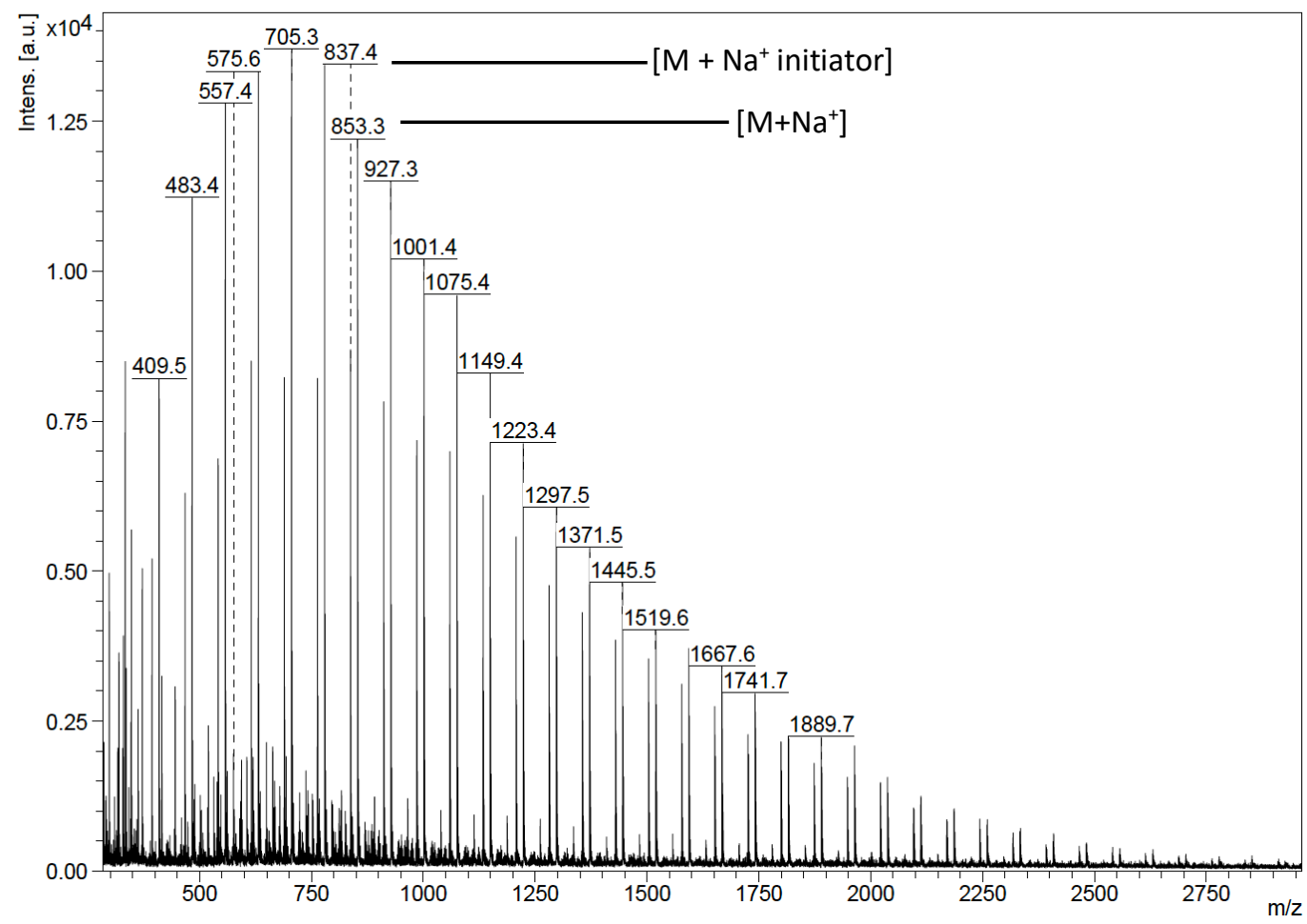

Figure S24. MALDI-TOF Mass Spectra HPG 5d 


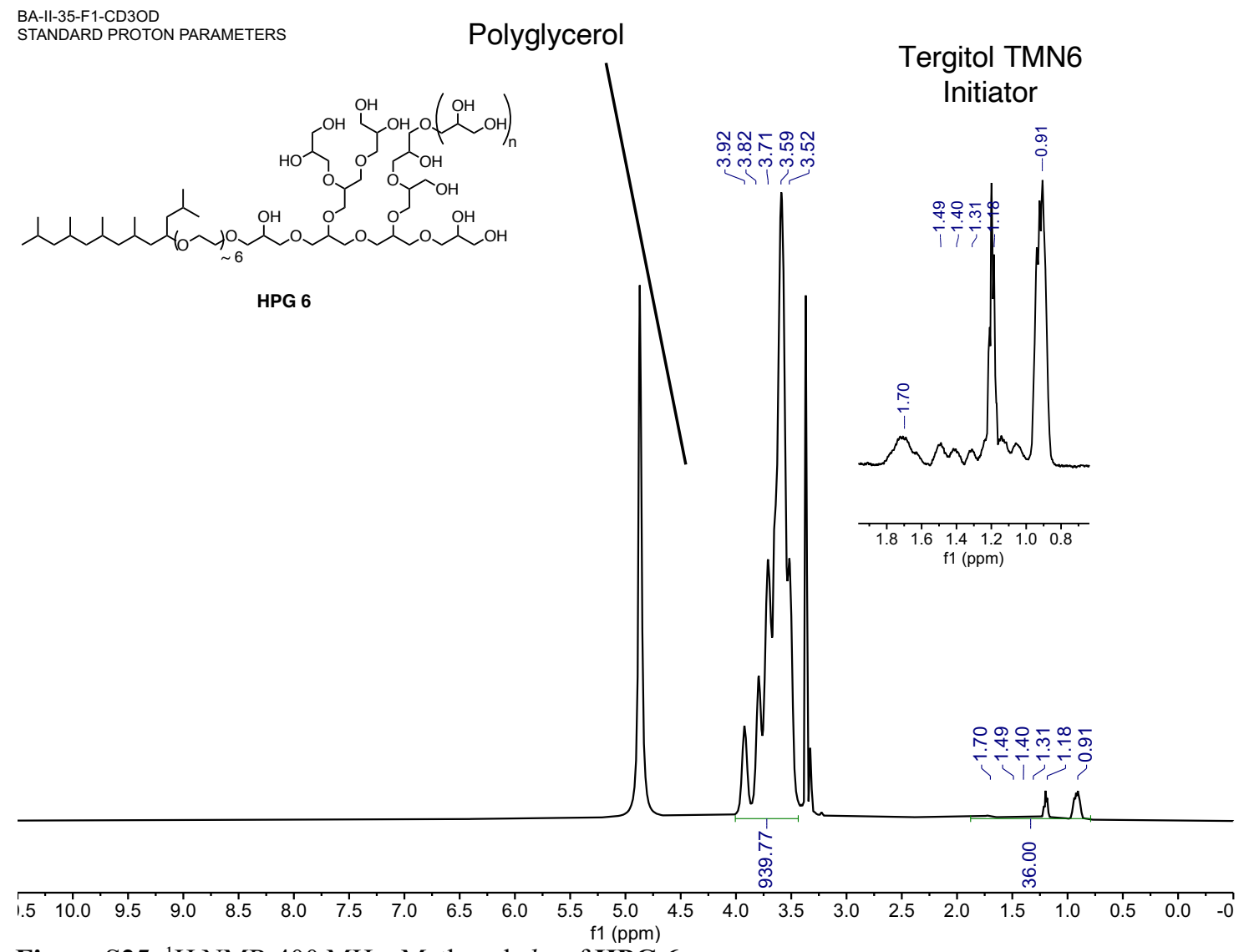

Figure S25. ${ }^{1} \mathrm{H}$ NMR $400 \mathrm{MHz}$, Methanol- $d_{4 i i}$ of HPG 6

13CNMR-BA-II-35-CD3OD-Crude

STANDARD CARBON PARAMETERS

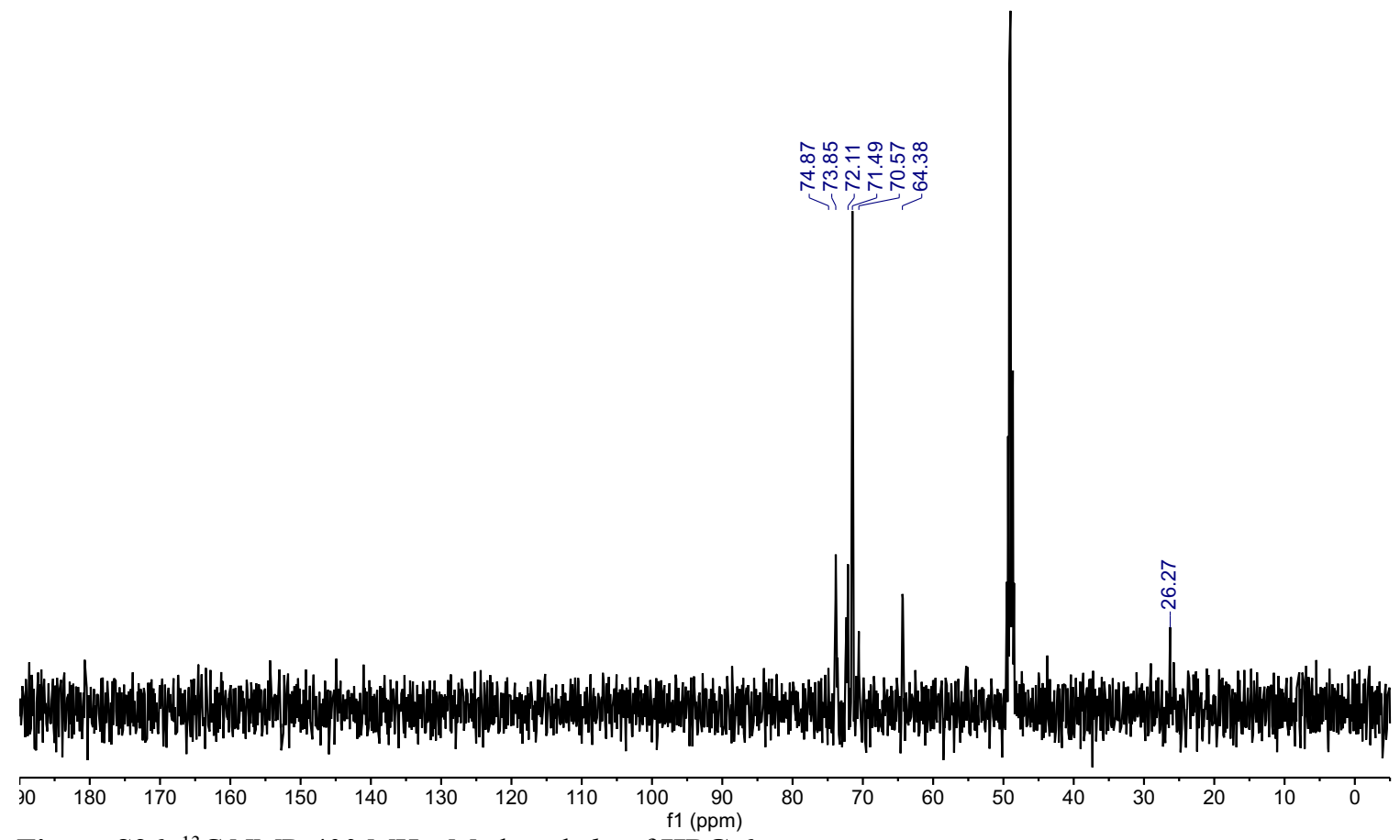

Figure S26. ${ }^{13} \mathrm{C}$ NMR $400 \mathrm{MHz}$, Methanol- $d_{4 i i}$ of HPG 6 


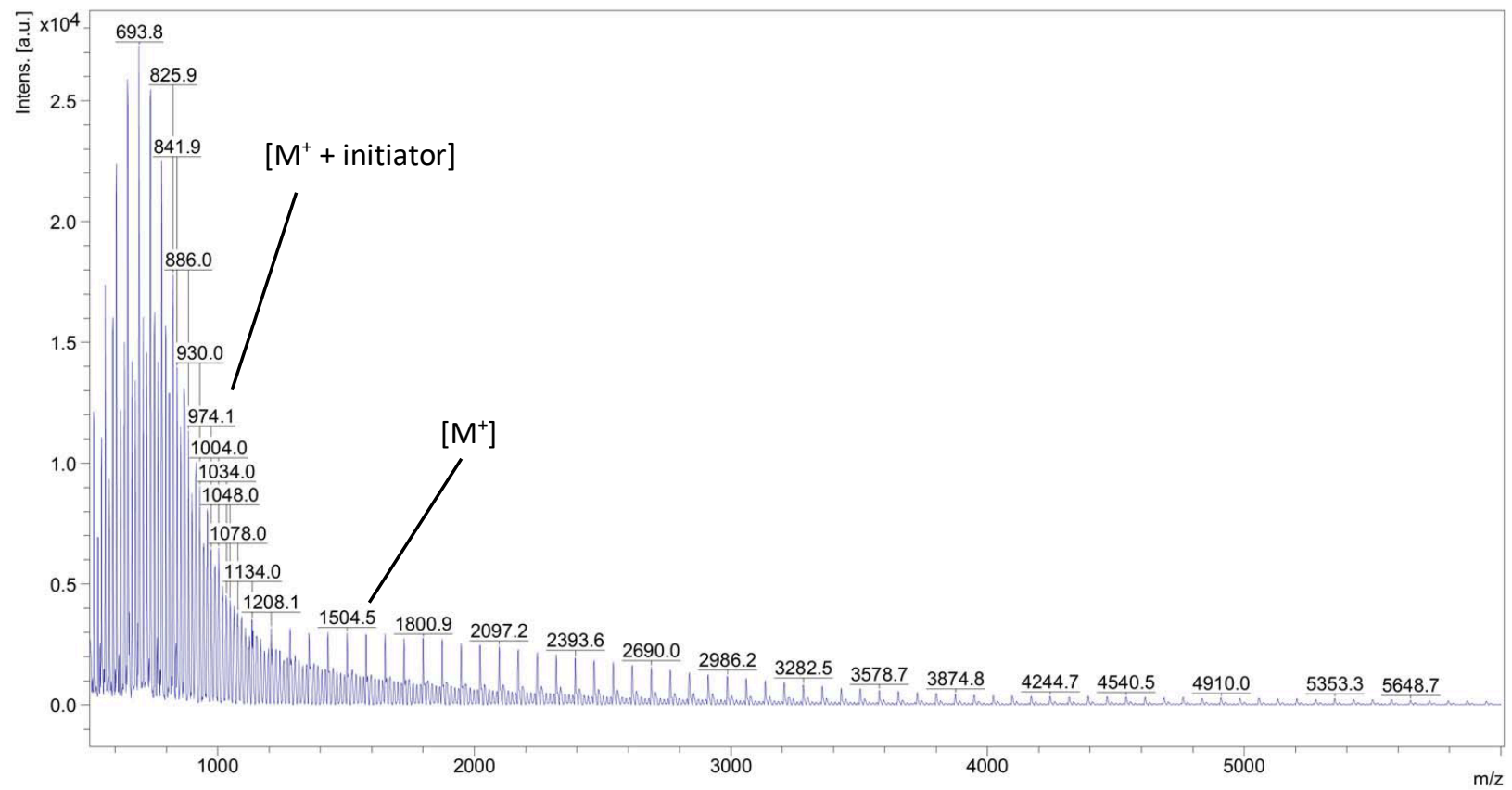

Figure S27. MALDI-TOF Mass Spectra HPG 6

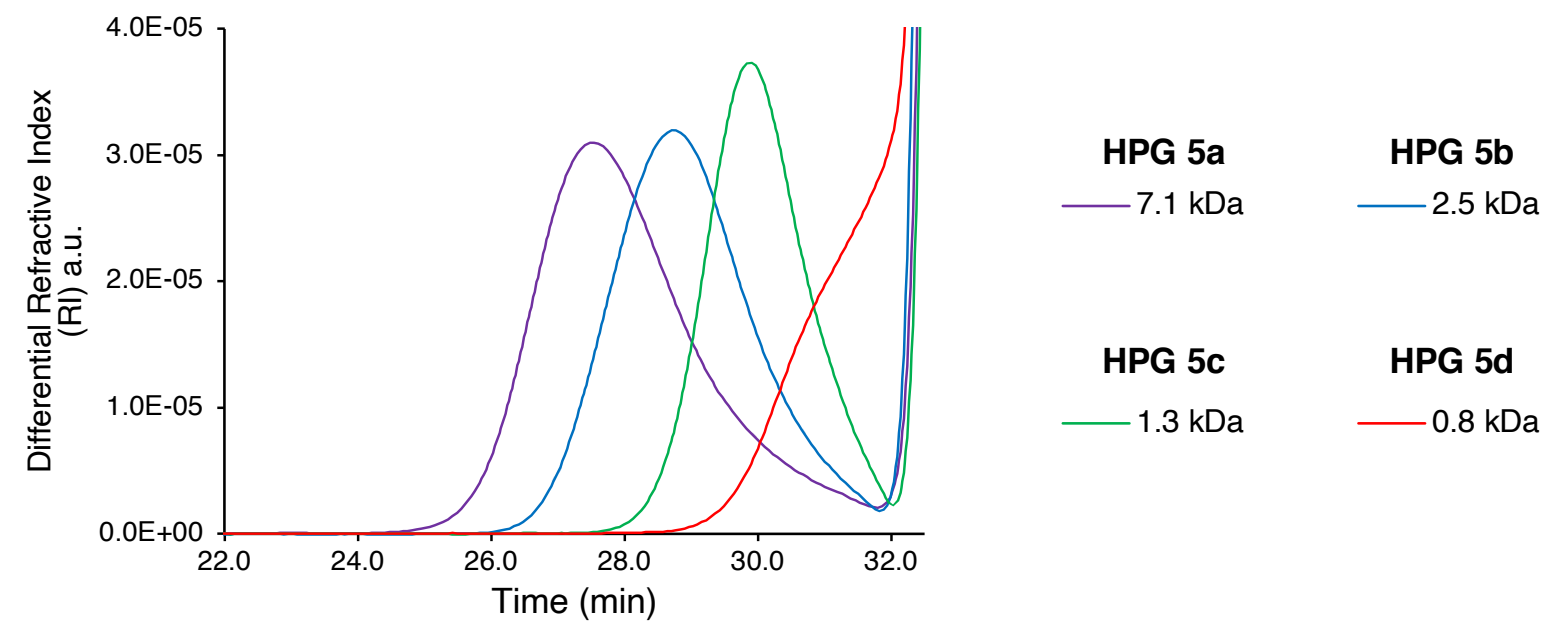

Figure S28. SEC-MALS trace of HPG 5a-d. 

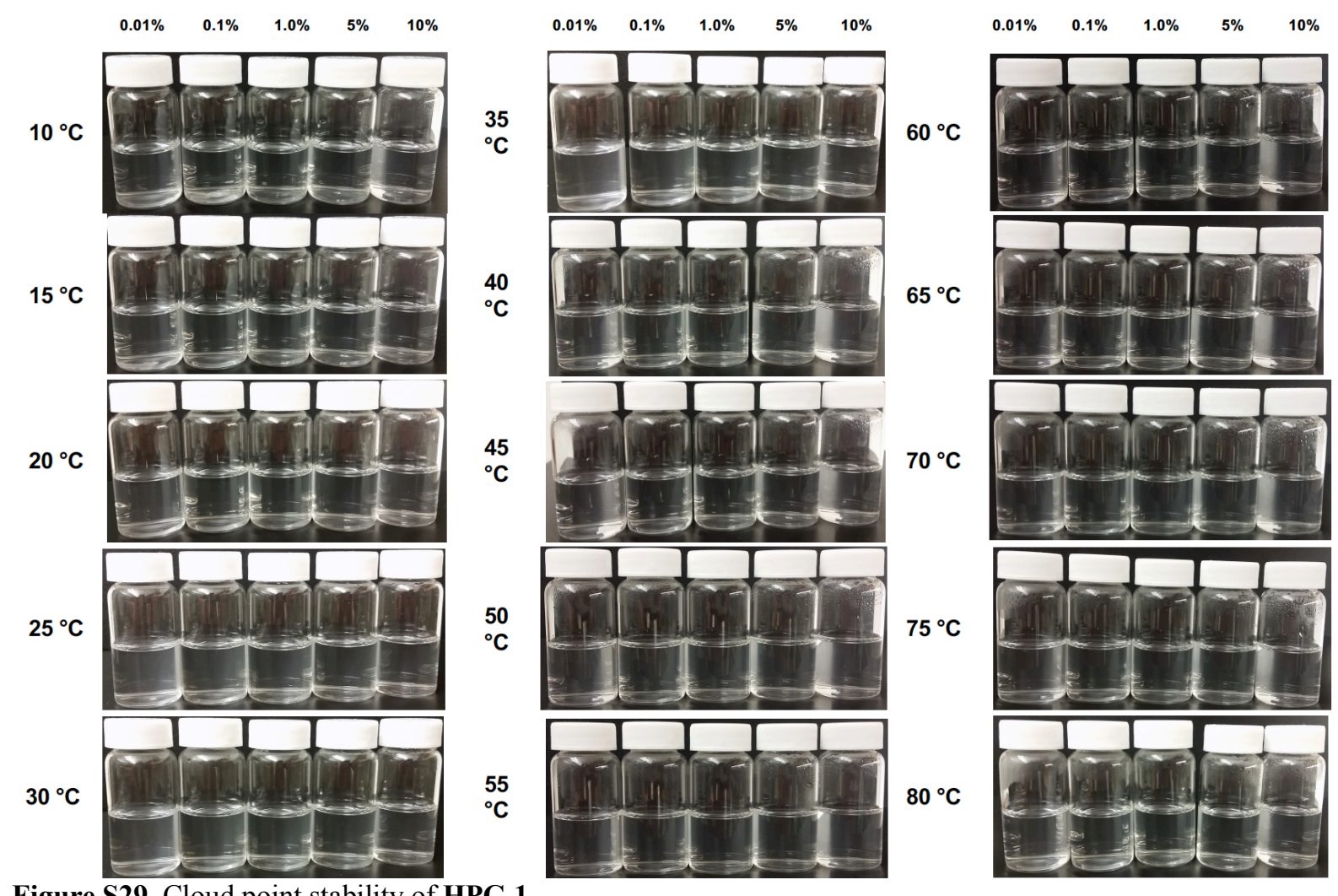

Figure S29. Cloud point stability of HPG 1
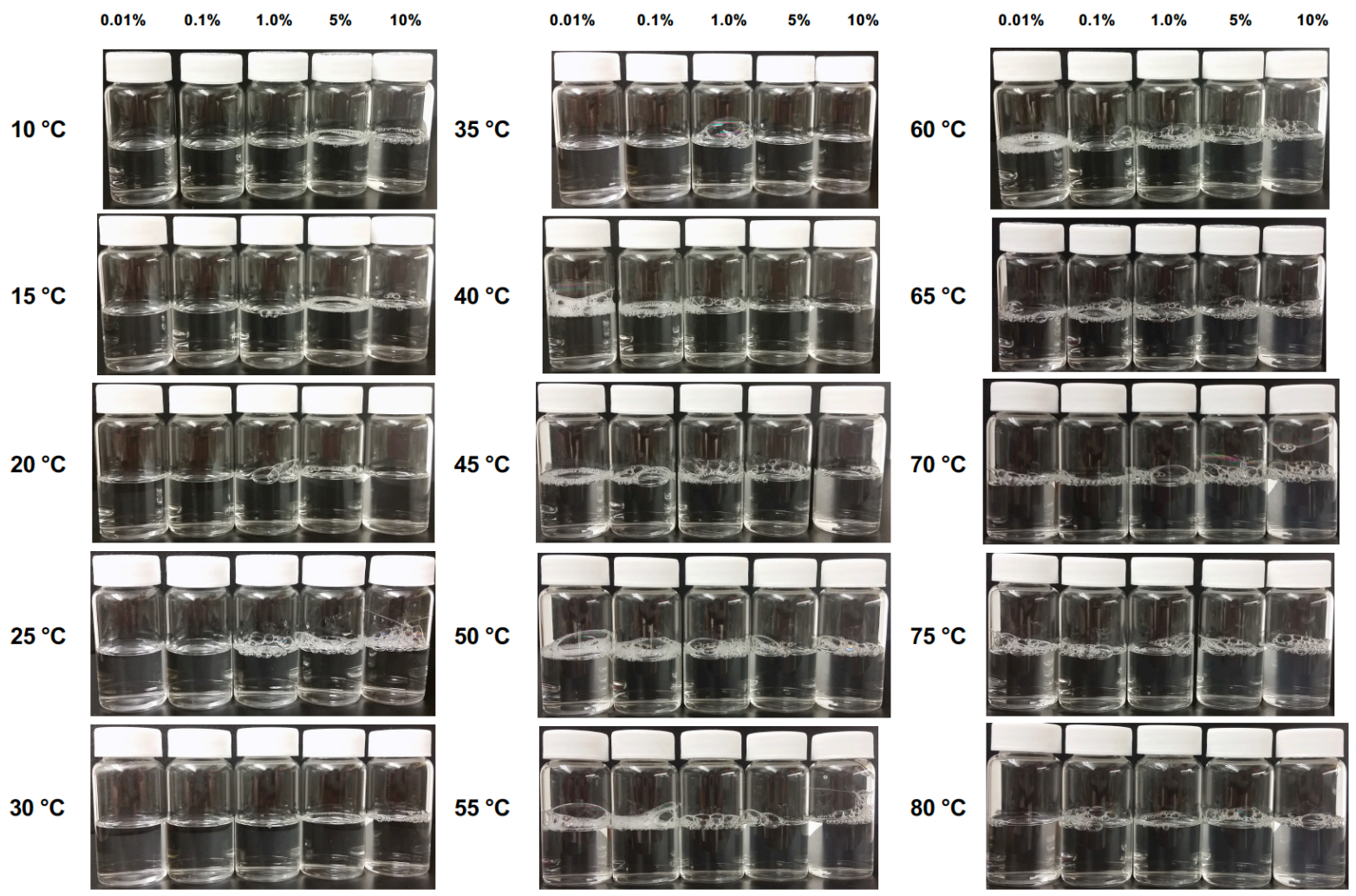

Figure S30. Cloud point stability of HPG 6 

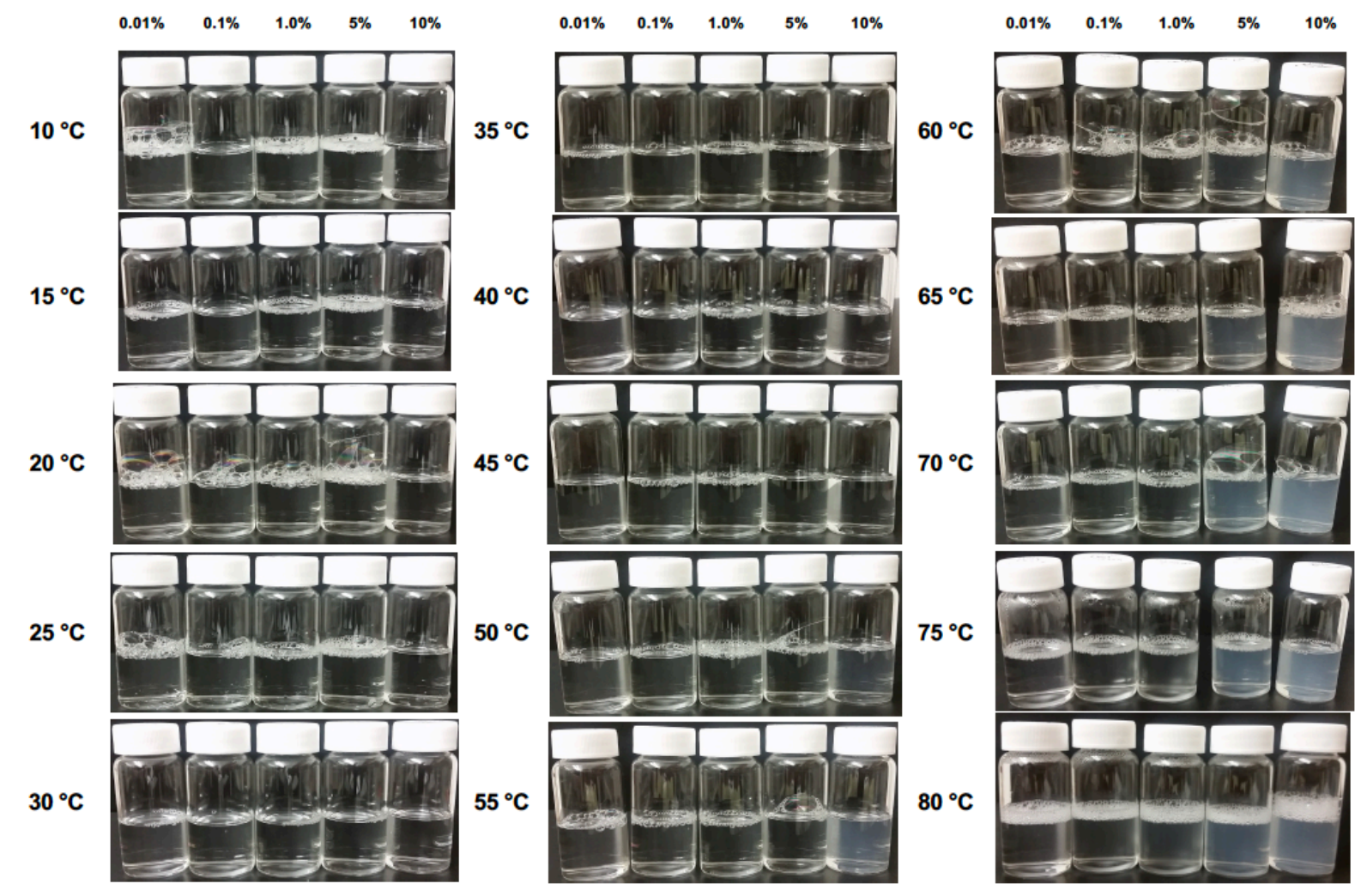

Figure S31. Cloud point stability of HPG 5d.
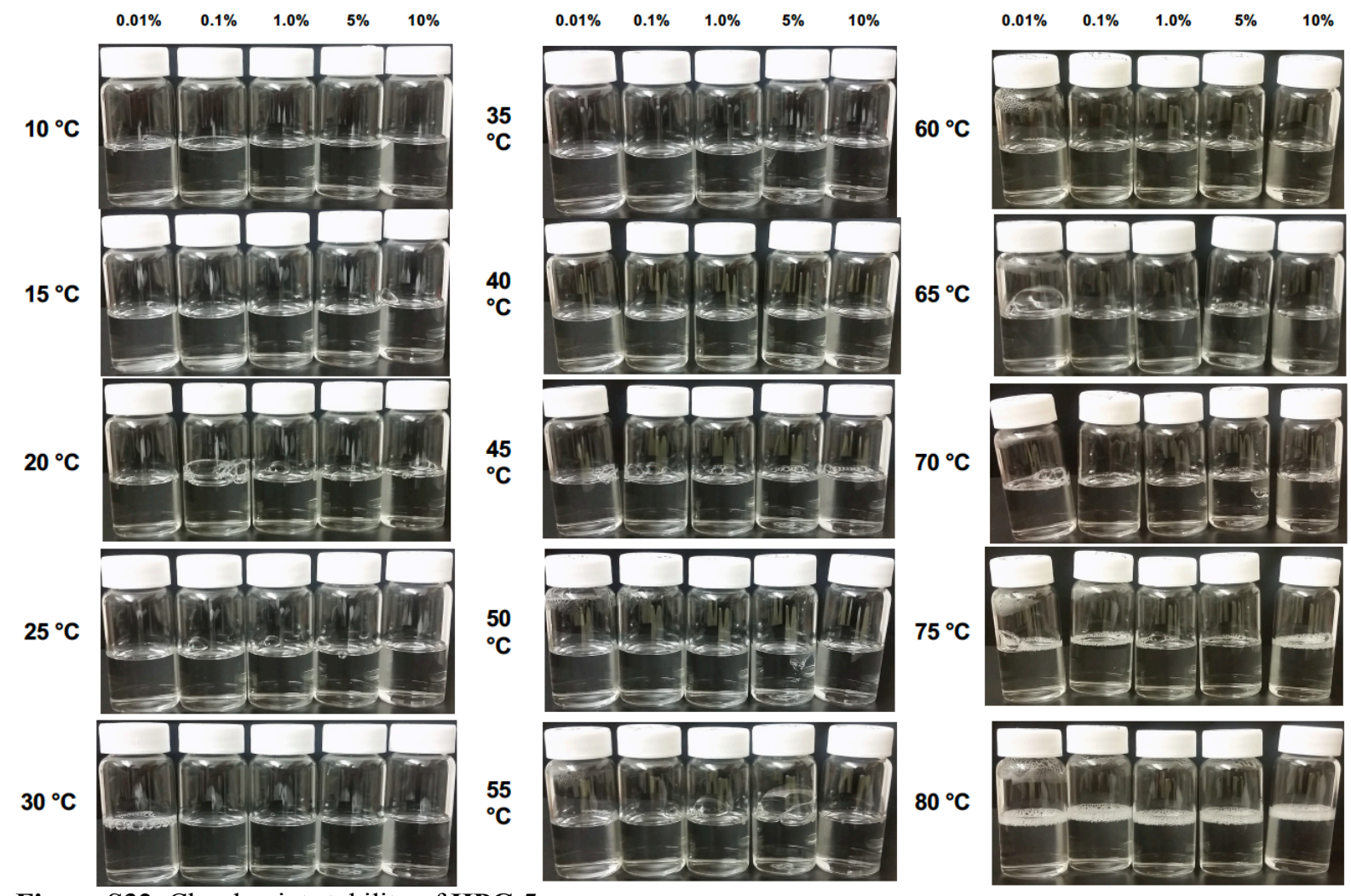

Figure S32. Cloud point stability of HPG 5c. 

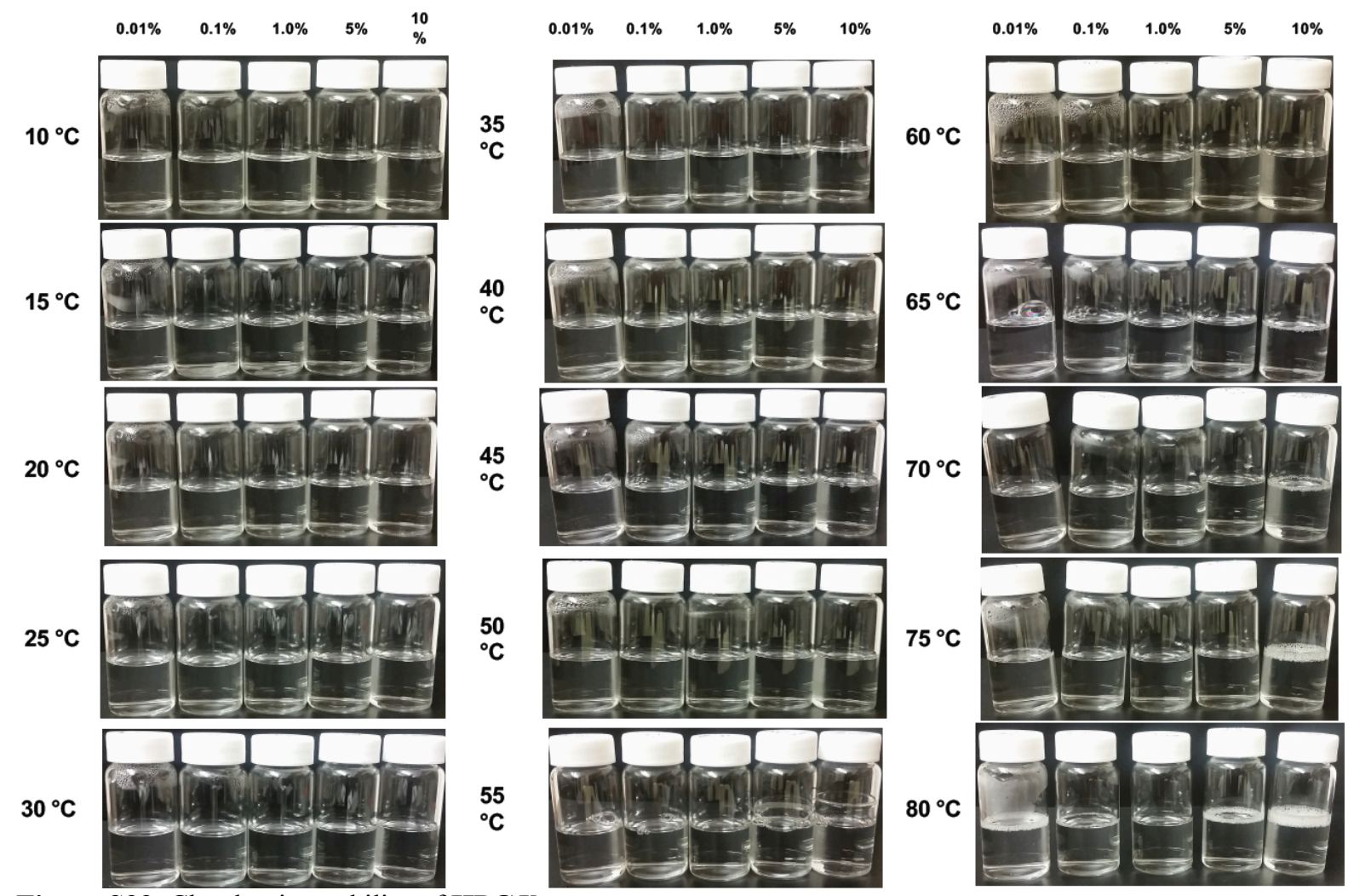

Figure S33. Cloud point stability of HPG5b.
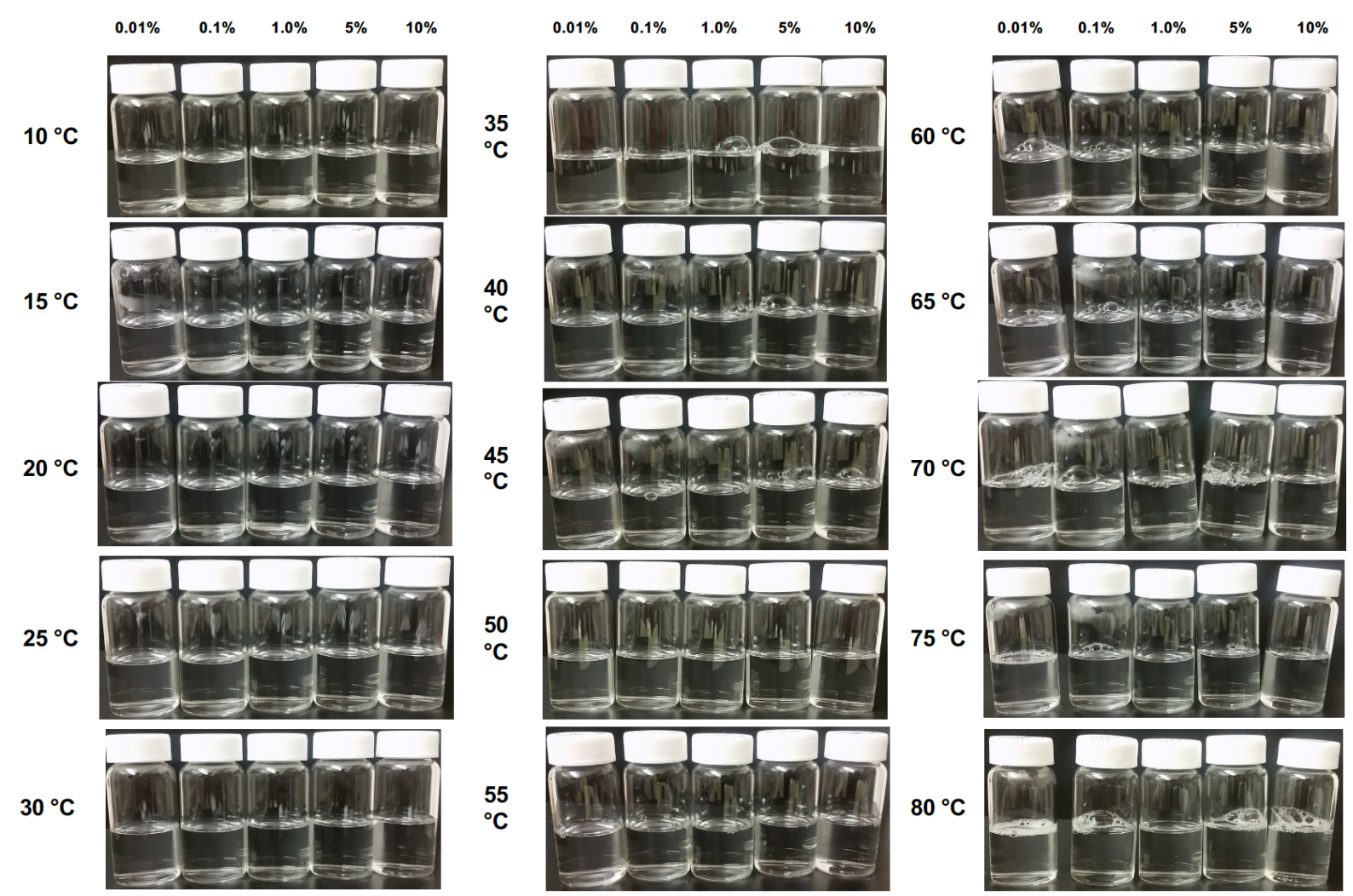

Figure S34. Cloud point stability of HPG 5a. 

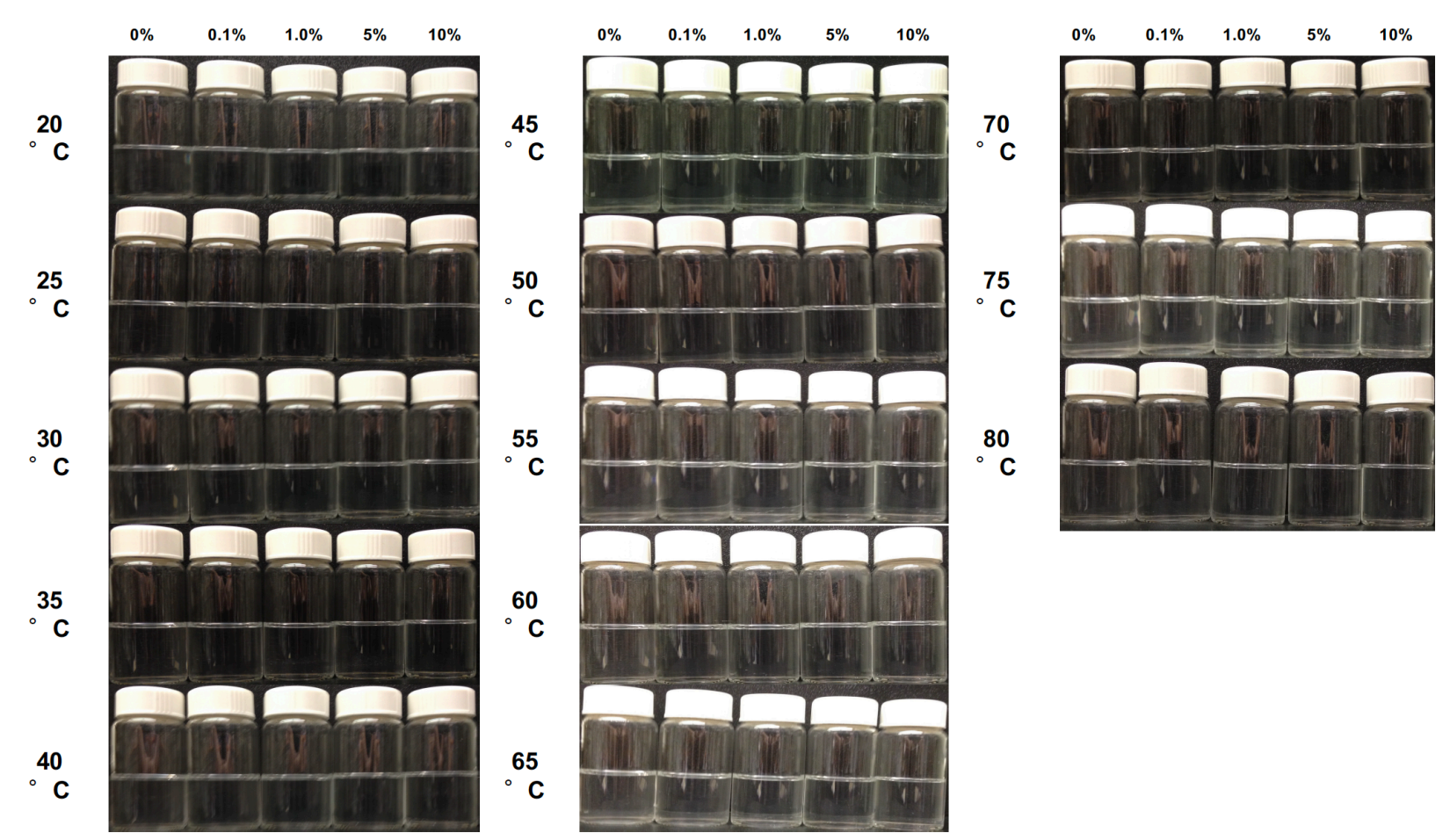

Figure S35. Cloud point stability of HPG 4.
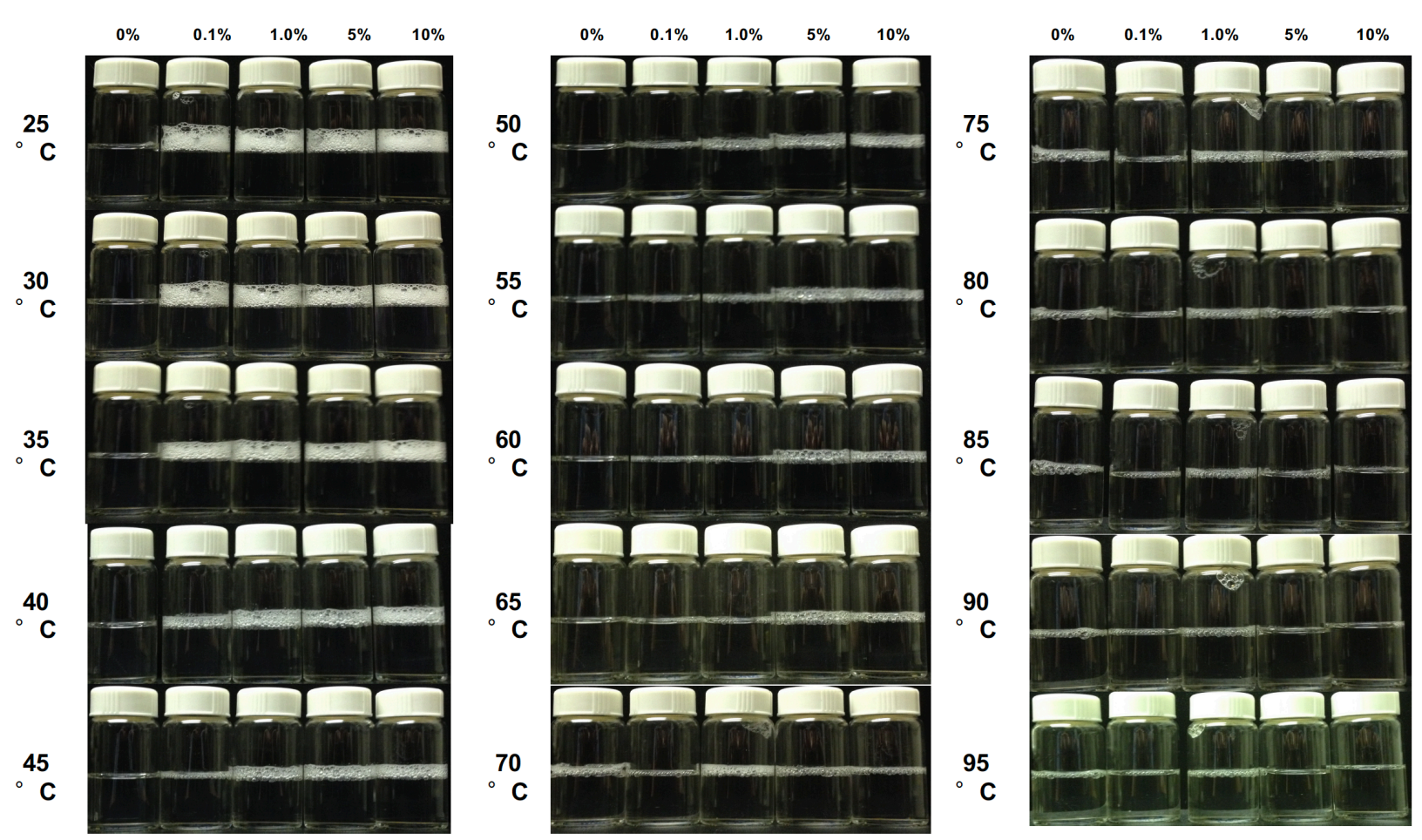

Figure S36. Cloud point stability of HPG 2. 


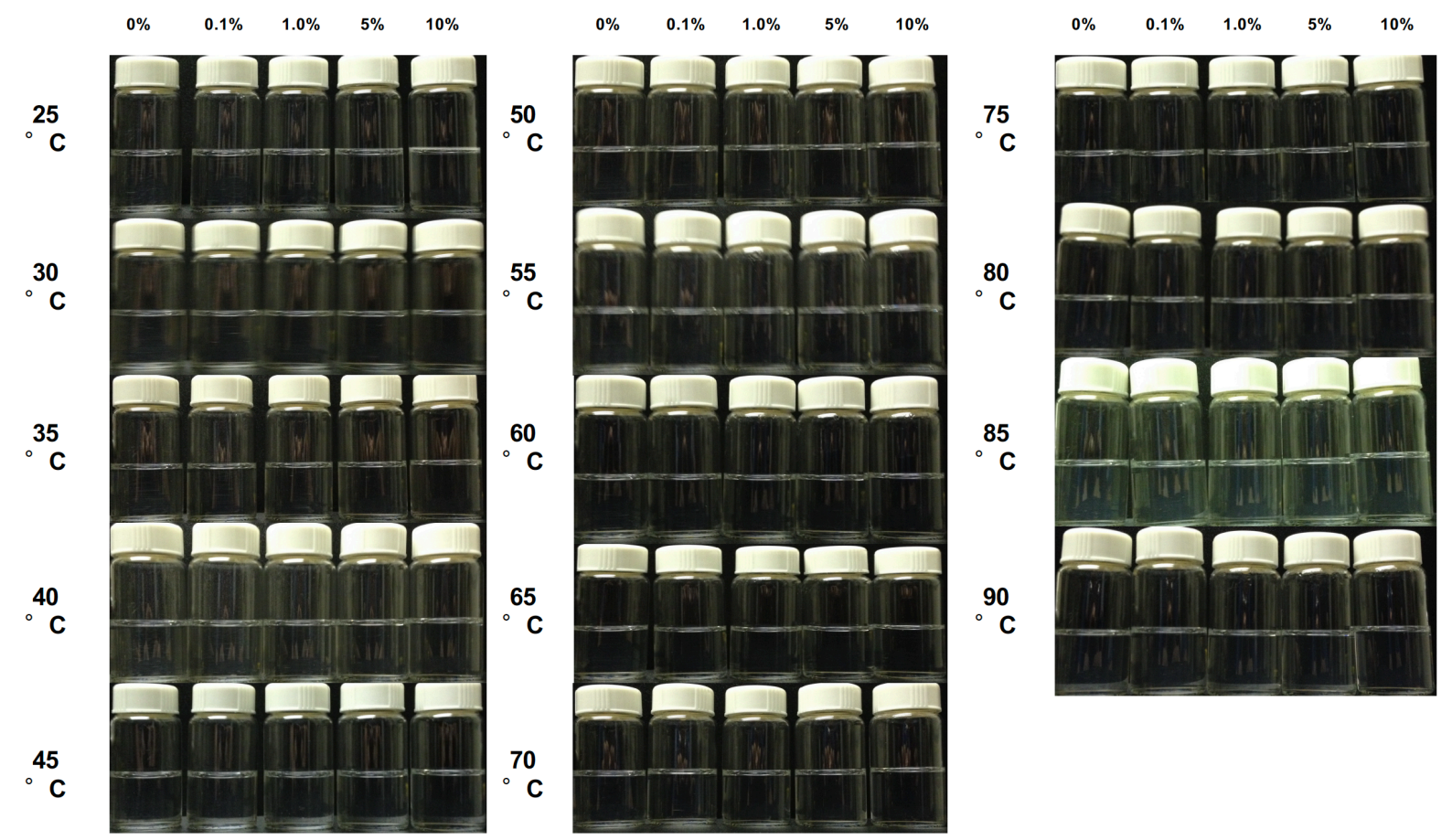

Figure S37. Cloud point stability of HPG 2. 

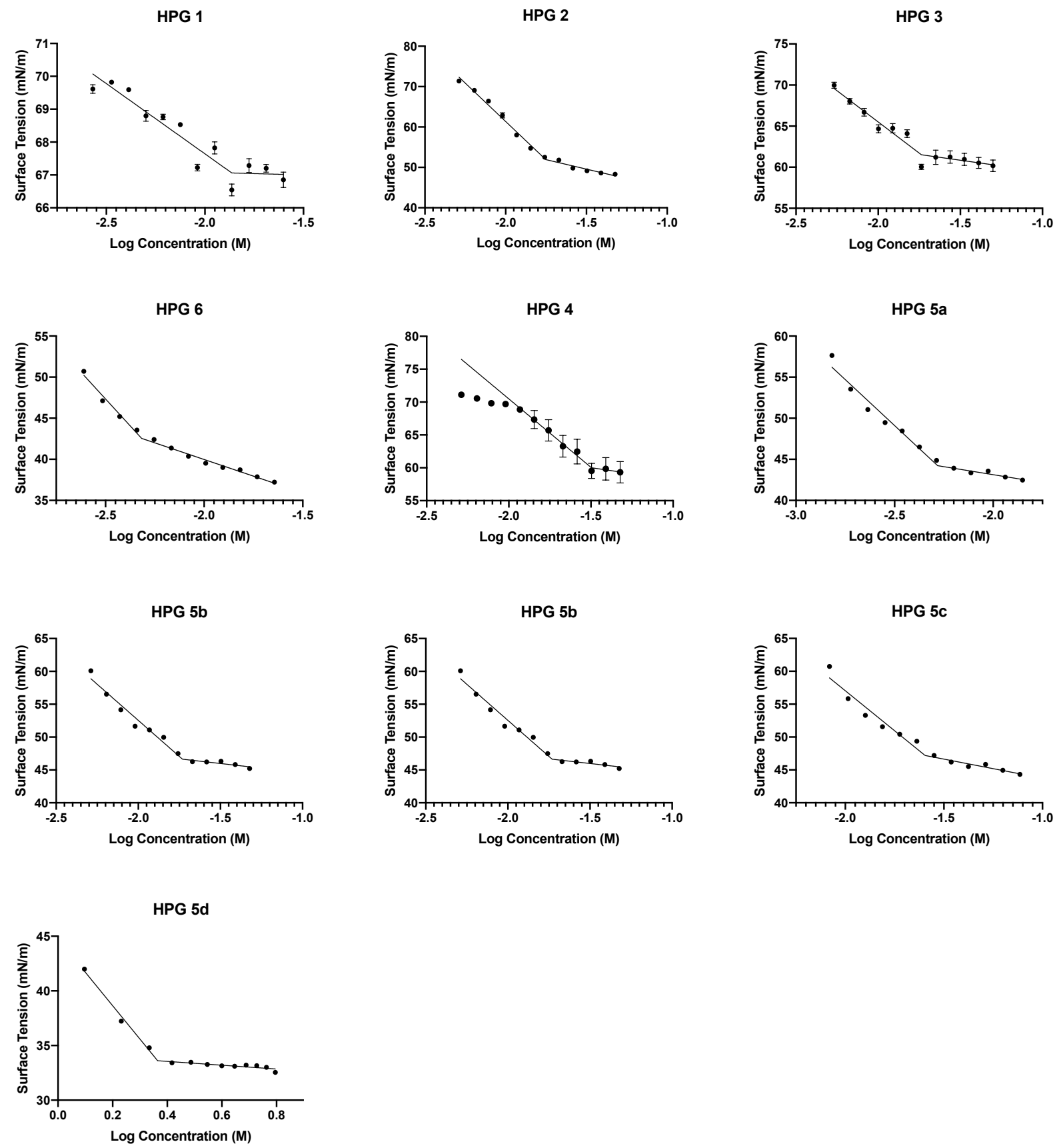

Figure S38. Surface tension versus $\log C$ with segmental linear regression fit for the determination of CMC, maximum surface excess at $\mathrm{CMC}\left(\Gamma_{\max }\right)$, and minimum area per molecule at interface $\left(\mathrm{A}_{\min }\right)$. 